\title{
MIMO Multiple Access Channel With an Arbitrarily Varying Eavesdropper: Secrecy Degrees of Freedom
}

\author{
Xiang He, Member, IEEE, Ashish Khisti, Member, IEEE, and Aylin Yener, Member, IEEE
}

\begin{abstract}
A two-transmitter Gaussian multiple access wiretap channel with multiple antennas at each of the nodes is investigated. The channel matrices of the legitimate users are fixed and revealed to all the terminals, whereas the channel matrices of the eavesdropper are arbitrarily varying and only known to the eavesdropper. The secrecy degrees of freedom (s.d.o.f.) region under a strong secrecy constraint is characterized. A transmission scheme that orthogonalizes the transmit signals of the two users at the intended receiver, and uses a single-user wiretap code for each user, is shown to achieve the s.d.o.f. region. The converse involves establishing an upper bound on a weighted-sum-rate expression. This is accomplished by using induction, where at each step one combines the secrecy and multiple-access constraints associated with an adversary eavesdropping a carefully selected group of sub-channels.
\end{abstract}

Index Terms - Arbitrarily varying channel, information theoretic security, MIMO multiple access wiretap channel, secrecy degrees of freedom.

\section{INTRODUCTION}

I NFORMATION theoretic security was first introduced by Shannon in [1], which studied the problem of transmitting confidential information in a communication system in the presence of an eavesdropper with unbounded computational power. Since then, an extensive body of work has been devoted to studying this problem for different network models by deriving fundamental transmission rate limits [2]-[4] and designing lowcomplexity schemes to approach these limits in practice [5], [6].

Secure communication using multiple antennas was extensively studied as well, see e.g., [7]-[18], [30]. These works investigated efficient signaling mechanisms using the spatial degrees of freedom provided by multiple antennas to limit an eavesdropper's ability to decode information. The underlying information theoretic problem, the multiantenna wiretap channel, was studied and the associated secrecy capacity

Manuscript received February 24, 2012; revised January 17, 2013; accepted March 18, 2013. Date of publication June 20, 2013; date of current version July 10, 2013. This work was supported in part by NSF Grant 0964362. A. Khisti was supported by an NSERC Discovery Grant. This paper was presented in part at the 49th Annual Allerton Conference on Communication, Control, and Computing, Monticello, IL, September, 2011.

$\mathrm{X}$. He was with the Department of Electrical Engineering, Pennsylvania State University, University Park, PA 16802 USA. He is now with Microsoft, Redmond, WA98052 USA (e-mail: xianghe@microsoft.com).

A. Khisti is with the Department of Electrical and Computer Engineering, University of Toronto, Toronto, ON M5S 3G4 Canada (e-mail: akhisti@comm. utoronto.ca).

A. Yener is with the Department of Electrical Engineering, Pennsylvania State University, University Park, PA 16802 USA (e-mail: yener@ee.psu.edu). Communicated by T. Uyematsu, Associate Editor for Shannon Theory.

Digital Object Identifier 10.1109/TIT.2013.2256952 was identified. We note that these works assumed that the eavesdropper's channel state information is available either completely or partially, although such an assumption may not be justified in practice.

As a more pessimistic but stronger assumption, references [19]-[21] study secrecy capacity when the eavesdropper channel is arbitrarily varying and its channel states are known to the eavesdropper only. Reference [20] studies the single-user Gaussian multi-input-multi-output (MIMO) wiretap channel and characterizes the secrecy degrees of freedom (s.d.o.f.). The same paper extended the single user analysis to the two users Gaussian MIMO multiple access (MIMO-MAC) channel and characterized the s.d.o.f. region when all the legitimate terminals had equal number of antennas. However, the MIMO-MAC with arbitrary number of antennas at the terminals was left as an open problem.

Our main contribution is to fully characterize the s.d.o.f. region of the two-transmitter MIMO MAC channel when the eavesdropper channel is arbitrarily varying. We show that the s.d.o.f. region can be achieved by a scheme that orthogonalizes the transmit signals of the two users at the intended receiver. Moreover, it suffices to use a single-user wiretap channel code [20] and no coordination between the users is necessary except for synchronization and sharing the transmit dimensions. To establish the optimality of this scheme, our converse proof decomposes the MIMO MAC channel into a set of parallel and independent channels using the generalized singular value decomposition (GSVD). A set of eavesdroppers, each monitoring a subset of links, is selected using an induction procedure and the resulting secrecy constraints are combined to obtain an upper bound on a weighted sum-rate expression. The outer bound matches the achievable rate in terms of the s.d.o.f. region, thus settling the open problem raised in [20] for the case of two transmitters.

The scalar multiple-access channel when the eavesdropper channel is perfectly known, has been studied extensively e.g., [22]-[29]. If the channel model has real inputs and output, Gaussian signaling is in general suboptimal and user cooperating strategies, as well as signal alignment techniques, are necessary [26]. It was shown in [28, Sec. 5.16] that the individual s.d.o.f. of this model could not exceed 2/3. Recently, [29] improves this result and shows that the sum s.d.o.f. of this model cannot exceed $2 / 3$ and shows this is achievable for almost all channel gains by using a scheme that transmits a superposition of information and noise symbols. Interference alignment is used to align the noise symbols at the legitimate receiver, and simultaneously mask the information symbols at the eavesdropper. 
The remainder of this paper is organized as follows. In Section II, we describe the system model. The main result is stated as Theorem 1 in Section IV. The proof of the theorem is divided into two parts. First, we establish the result for the case of parallel channels in Section V. Subsequently, in Section VI we establish the result for the general case by decomposing the MIMO-MAC channel into a set of independent parallel channels. Such a reduction is used both in the proof of the converse as well as the coding scheme. Section VII concludes the paper.

We use the following notation throughout the paper. For a set $\mathcal{A}, V_{\mathcal{A}}$ denotes the set of random variables $\left\{V_{j}, j \in \mathcal{A}\right\}$ and similarly $V_{i, \mathcal{A}}$ denotes the set of variables $\left\{V_{i, j}, j \in \mathcal{A}\right\}$. We use $\left\{\delta_{n}\right\}$ to denote a nonnegative sequence of $n$ that converges to 0 when $n$ goes to $\infty$. We use bold uppercase font for matrices and vectors. The distinction between matrices and vectors will be clear from the context. For a set $\mathcal{A},|\mathcal{A}|$ denotes its cardinality and a short hand notation $x^{n}$ is used for the sequence $\left\{x_{1}, x_{2}, \ldots, x_{n}\right\}$. Finally, $\phi$ denotes the empty set.

\section{SYSTEM MODEL}

As shown in Fig. 1, we consider a discrete-time channel model where two transmitters communicate with one receiver in the presence of an eavesdropper. We assume transmitter $i$ has $N_{T_{i}}$ antennas, $i=1,2$, the legitimate receiver has $N_{R}$ antennas whereas the eavesdropper has $N_{E}$ antennas. The channel model is given by

$$
\begin{aligned}
& \mathbf{Y}(i)=\sum_{k=1}^{2} \mathbf{H}_{k} \mathbf{X}_{k}(i)+\mathbf{Z}(i) \\
& \tilde{\mathbf{Y}}(i)=\sum_{k=1}^{2} \tilde{\mathbf{H}}_{k}(i) \mathbf{X}_{k}(i)
\end{aligned}
$$

where $i \in\{1, \ldots, n\}$ denotes the time-index, $\mathbf{H}_{k}, k=1,2$, are channel matrices and $\mathbf{Z}$ is the additive Gaussian noise observed by the intended receiver, which is composed of independent rotationally invariant complex Gaussian random variables with zero mean and unit variance. The sequence of eavesdropper channel matrices $\left\{\tilde{\mathbf{H}}_{k}(i), k=1,2\right\}$, is an arbitrary sequence of length $n$ and only revealed to the eavesdropper. In contrast, $\mathbf{H}_{k}, k=1,2$ are revealed to both the legitimate parties and the eavesdropper and remain constant during the period of communication. We assume $N_{E}$, the number of eavesdropper antennas, is known to the legitimate parties and the eavesdropper.

We define a length $n$ code $\mathcal{C}^{(n)}$ for our setup as follows. User $k, k=1,2$, wishes to transmit a confidential message $W_{k}, k=$ 1,2 , to the receiver over $n$ channel uses, while both messages, $W_{1}$ and $W_{2}$, must be kept confidential from the eavesdropper. The message $W_{1}$ and $W_{2}$ are uniformly distributed over the sets $\mathcal{W}_{1}$ and $\mathcal{W}_{2}$, respectively. We assume that $\left|\mathcal{W}_{k}\right|=2^{n R_{s, k}}$. User $k$ transmits an input sequence $\mathbf{X}_{k}^{n}=f_{k, n}\left(W_{k}\right)$ where $f_{k, n}: \mathcal{W}_{k} \rightarrow \mathbb{C}^{n}$ is the encoding function at user $k$. The decoder outputs an estimate $\left(\hat{W}_{1}, \hat{W}_{2}\right)=g_{n}\left(\mathbf{Y}^{n}\right)$ of the transmitted messages where $g_{n}: \mathbb{C}^{n} \rightarrow \mathcal{W}_{1} \times \mathcal{W}_{2}$ is the decoding function. The error probability is defined as, $\operatorname{Pe}^{(n)}=\operatorname{Pr}\left(\hat{W}_{1} \neq\right.$ $\left.W_{1} \cup \hat{W}_{2} \neq W_{2}\right)$.

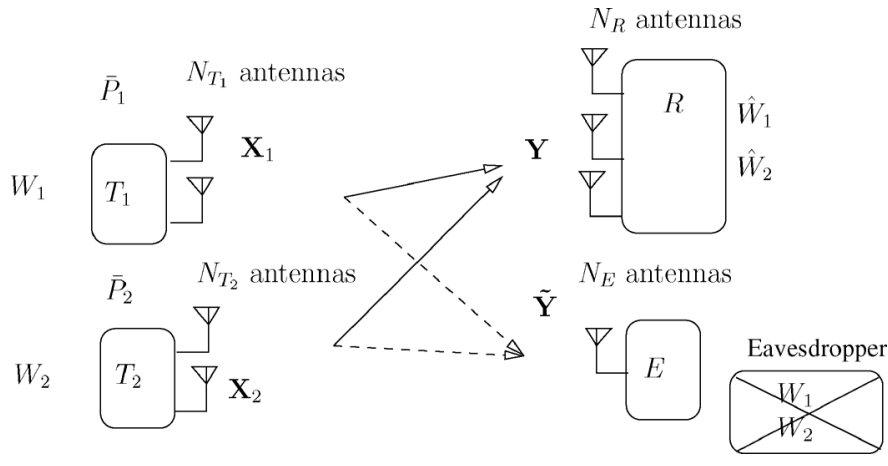

Fig. 1. The MIMO MAC wiretap channel where $N_{T_{1}}=N_{T_{2}}=2, N_{R}=$ $3, N_{E}=1$.

A sequence of codes $\mathcal{Q}=\left\{\mathcal{C}^{(n)}\right\}$ is said to be feasible if the following conditions are satisfied.

a) Reliability Constraint: $\lim _{n \rightarrow \infty} \mathrm{Pe}^{(n)}=0$

b) Power Constraint: The sequence $\mathbf{X}_{k}^{n}$ must satisfy the power constraint

$$
\lim _{n \rightarrow \infty} \frac{1}{n} \sum_{i=1}^{n}\left|\mathbf{X}_{k}(i)\right|^{2} \leq \bar{P}_{k}, \quad k=1,2,
$$

for each message $W_{1} \in \mathcal{W}_{1}$ and $W_{2} \in \mathcal{W}_{2}$.

c) Secrecy Constraint: We consider the strong secrecy constraint [20]

$\lim _{n \rightarrow \infty} \sup _{\left\{\tilde{H}_{k}^{n}, k=1,2\right\}} I\left(W_{1}, W_{2} ; \tilde{\mathbf{Y}}^{n} \mid \tilde{\mathbf{H}}_{k}^{n}=\tilde{\mathbf{H}}_{k}^{n}, k=1,2\right)=0$.

We say that the rate-pair $\left(R_{s, 1}, R_{s, 2}\right)$ is achievable if there exists a sequence of codes $\mathcal{Q}$ with $|\mathcal{W}|_{k}=2^{n R_{s, k}}$ that satisfies the above conditions. The associated s.d.o.f. are defined as [30], [31]:

$$
d_{k}=\limsup _{\bar{P}_{k} \rightarrow \infty} \frac{R_{s, k}\left(\bar{P}_{k}\right)}{\log _{2} \bar{P}_{k}} .
$$

The set of all achievable s.d.o.f. constitutes secrecy degrees of freedom. We note the use of lim sup in (5) implies that for any coding scheme one must consider a subsequence of powers that attains the lim sup. For the upper and lower bounds we consider, the limit actually exists, and hence lim sup and lim are the same.

We make the following additional remark about the channel model.

Remark 1: An arbitrarily varying channel (AVC) is defined by a stochastic mapping $W^{n}: \mathcal{X}^{n} \rightarrow \mathcal{Y}^{n}$, where $\mathcal{X}$ and $\mathcal{Y}$ are the alphabets of the channel input and output symbols, respectively, and

$$
W^{n}\left(y^{n} \mid x^{n}, s^{n}\right)=\prod_{i=1}^{n} W\left(y_{i} \mid x_{i}, s_{i}\right) .
$$

Here $W(y \mid x, s)$ is the transition probability that the channel output symbol $y$ is observed when a channel input symbol $x$ is transmitted and the channel state equals $s$. The sequence $s^{n}=$ $\left(s_{1}, s_{2}, \ldots, s_{n}\right)$ denotes the sequence of channel states that can vary in an arbitrary manner. There is a large variety of problems on AVC channels depending on the nature of the error-criteria used (average or maximal error) and the permissible coding 


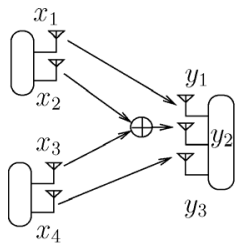

(a)

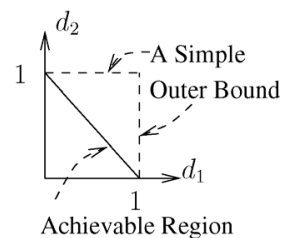

(b)
Fig. 2. (a) A special case of MIMO MAC wiretap channel where $N_{T_{1}}=$ $N_{T_{2}}=2, N_{R}=3, N_{E}=1$, (b) Comparison between achievable s.d.o.f. region and a simple outer bound derived by considering one eavesdropper at a time.

strategies (deterministic versus random coding). We refer the reader to [32, Ch. 12] for a comprehensive treatment of AVC channel models.

In this paper, we assume that the eavesdropper's channel is an AVC channel, where the state variable $s(i)=\left(\tilde{\mathbf{H}}_{1}(i), \tilde{\mathbf{H}}_{2}(i)\right)$ [cf. (2)]. We assume that no common randomness is shared between the legitimate users. The encoders are allowed to use private randomness in their encoding functions. Furthermore, we assume that the state sequence be independent of $\mathbf{X}^{n}$ :

$$
\operatorname{Pr}\left(\mathbf{X}^{n}=\mathbf{x}^{n} \mid \tilde{\mathbf{H}}_{1}^{n}=\tilde{\mathbf{H}}_{1}^{n}, \tilde{\mathbf{H}}_{2}^{n}=\tilde{\mathbf{H}}_{2}^{n}\right)=\operatorname{Pr}\left(\mathbf{X}^{n}=\mathbf{x}^{n}\right) .
$$

The evaluation of, e.g., (4) is based on this condition.

Remark 2: For proving our converse it is sufficient to consider a (weaker) compound channel model [30] - the channel matrices $\tilde{\mathbf{H}}_{k}$ are selected at the start of the communication from a certain set, say $\mathcal{H}$, and remain fixed for the entire codeword. By a judicious choice of the set $\mathcal{H}$, it is possible to obtain a matching upper bound for the s.d.o.f.

\section{Motivation}

We discuss a simple example that illustrates why the problem considered is nontrivial. As illustrated in Fig. 2(a), in this example, each transmitter has two antennas and the intended receiver has three antennas, while the eavesdropper has only one antenna. Let $x_{1}, x_{2}, x_{3}, x_{4}$ denote the transmitted signals from the two users and $y_{1}, y_{2}, y_{3}$ denote the signals observed by the intended the receiver. And the main channel is given by

$$
\begin{aligned}
& y_{1}=x_{1}+z_{1}, \quad y_{3}=x_{4}+z_{3} \\
& y_{2}=x_{2}+x_{3}+z_{2}
\end{aligned}
$$

where $z_{i}, i=1,2,3$ denote additive channel noise. As shown in [20], a s.d.o.f. $\min \left(N_{T_{k}}, N_{R}\right)-N_{E}=1$ is achievable for a user if the other user remains silent. Time sharing between these two users leads to the following achievable s.d.o.f. region:

$$
d_{1}+d_{2} \leq 1, \quad d_{k} \geq 0, \quad k=1,2 .
$$

\section{A. Cut-Set Upper Bound}

For the converse, we begin by considering a simple "cut-set" like upper bound, which reduces each channel to a single-user
MIMO wiretap channel. First, by revealing the signals transmitted by user 2 to the intended receiver and assuming that the eavesdropper monitors either $x_{1}$ or $x_{2}$ we have that $d_{1} \leq 1$. Similarly, we argue that $d_{2} \leq 1$. To obtain an upper bound on the sum-rate, we let the two transmitters to cooperate and reduce the system to a $3 \times 3$ MIMO link. The s.d.o.f. of this channel [20] yields $d_{1}+d_{2} \leq 2$. This outer bound, illustrated in Fig. 2(b), does not match with the achievable region given by (9).

\section{B. Proposed Upper Bound}

As we shall show in Theorem 1, (9) is indeed the s.d.o.f. capacity region and hence a new converse is necessary to prove this result. It can be readily seen that the outer bound in Section III-A only considers one eavesdropper at a time. For example, when deriving $d_{1} \leq 1$, we assume there is only one eavesdropper which is monitoring either $x_{1}$ or $x_{2}$. When deriving $d_{2} \leq 1$, we assume there is only one eavesdropper which is monitoring either $x_{3}$ or $x_{4}$. Similarly, when deriving $d_{1}+d_{2} \leq 2$ we again assume that there is one eavesdropper on either of the links. Our key observation is that a tighter upper bound is possible to find if we consider the simultaneous effect of two eavesdroppers.

In our system model, because of the AVC model, there are infinitely many possible eavesdroppers, each corresponding to a different channel state sequence. The challenge is to find a finite number of eavesdroppers, whose joint effect leads to a tight converse. Our choice of eavesdroppers is based on the following intuition: when an eavesdropper chooses which links to monitor, it should give precedence to those links over which only one user can transmit. This is because these links are the major contributor to the sum s.d.o.f. $d_{1}+d_{2}$ since they are dedicated links to a certain user. Based on this intuition, we consider the following two eavesdroppers: one monitors $y_{1}$ for $W_{1}$ and the other monitors $y_{3}$ for $W_{2}$. As we shall show later in Lemma 1 , the first eavesdropper implies the following upper bound on $R_{1}$ :

$$
n\left(R_{1}-\delta_{n}\right) \leq I\left(x_{2}^{n} ; y_{2}^{n} \mid y_{1}^{n}, x_{\{3,4\}}^{n}\right)
$$

and the second eavesdropper implies the following upper bound on $R_{2}$ :

$$
n\left(R_{2}-\delta_{n}\right) \leq I\left(y_{1}^{n}, x_{\{3,4\}}^{n} ; y_{2}^{n}\right)
$$

Their joint effect can be captured by adding (10) and (11) [33], which leads to:

$$
n\left(R_{1}+R_{2}-2 \delta_{n}\right) \leq I\left(x_{2}^{n}, y_{1}^{n}, x_{\{3,4\}}^{n} ; y_{2}^{n}\right)
$$

Since there is only one term, which is $y_{2}^{n}$, at the right side of the mutual information $I\left(x_{2}^{n}, y_{1}^{n}, x_{\{3,4\}}^{n} ; y_{2}^{n}\right)$, we observe the sum s.d.o.f. cannot exceed 1, thereby justifying that (9) is indeed the largest possible s.d.o.f. region for Fig. 2(a).

As captured by (10) and (11), a simultaneous selection of two different eavesdroppers for the two users reduces the effective signal dimension at the receiver from three to one, thus leading to a tighter converse. As we shall show later in Section V-C, 


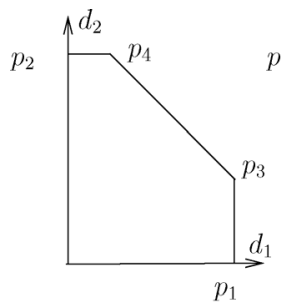

(a)

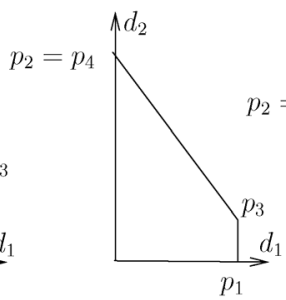

(b)

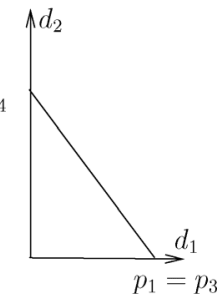

(c)
Fig. 3. The s.d.o.f. region in Theorem 1: (a) $0 \leq N_{E} \leq \min \left\{r_{0}-r_{1}, r_{0}-\right.$ $\left.r_{2}\right\}$, (b) $\min \left\{r_{0}-r_{1}, r_{0}-r_{2}\right\} \leq N_{E} \leq \max \left\{r_{0}-r_{1}, r_{0}-r_{2}\right\}$, (c) $\max \left\{r_{0}-r_{1}, r_{0}-r_{2}\right\} \leq N_{E}$.

in generalizing this example, we are required to systematically select a sequence of eavesdroppers using induction.

\section{MAIN RESULT}

In this section, we state the main result of this paper. To express our result, we define $r_{t}$ as the rank of $\mathbf{H}_{t}, t=1,2$ and $r_{0}$ as the rank of $\left[\mathbf{H}_{1} \mid \mathbf{H}_{2}\right]$. We will refer to $r_{t}$ as the number of transmit dimensions at user $t=1,2$ and $r_{0}$ as the number of dimensions at the receiver.

Theorem 1: The s.d.o.f. region of the MIMO multiple access channel with arbitrarily varying eavesdropper channel is given by the convex hull of the following five points of $\left(d_{1}, d_{2}\right)$ :

$$
\begin{aligned}
& p_{0}=(0,0) \\
& p_{1}=\left(\left[r_{1}-N_{E}\right]^{+}, 0\right) \\
& p_{2}=\left(0,\left[r_{2}-N_{E}\right]^{+}\right) \\
& p_{3}=\left(\left[r_{1}-N_{E}\right]^{+},\left[r_{0}-r_{1}-N_{E}\right]^{+}\right) \\
& p_{4}=\left(\left[r_{0}-r_{2}-N_{E}\right]^{+},\left[r_{2}-N_{E}\right]^{+}\right)
\end{aligned}
$$

where we use $[x]^{+} \triangleq \max \{x, 0\}$.

Fig. 3 illustrates the structure of the s.d.o.f. region as a function of the number of eavesdropping antennas. In Fig. 3(a), we have $N_{E} \leq \min \left(r_{0}-r_{1}, r_{0}-r_{2}\right)$. In this case, the s.d.o.f. region is a polymatroid [34, Definition 3.1] described by $d_{i} \leq r_{i}-N_{E}$ and $d_{1}+d_{2} \leq r_{0}-2 N_{E}$. Fig. 3(b) illustrates the shape of the s.d.o.f. region when $\min \left\{r_{0}-r_{1}, r_{0}-r_{2}\right\} \leq N_{E} \leq$ $\max \left\{r_{0}-r_{1}, r_{0}-r_{2}\right\}$. In Fig. 3(b), without loss of generality, we assume $r_{1}<r_{2}$ and the s.d.o.f. region is bounded by the lines $d_{i} \geq 0, d_{1} \leq r_{1}-N_{E}$ and

$$
\begin{aligned}
& \left(r_{1}+r_{2}-r_{0}\right) d_{1}+\left(r_{1}-N_{E}\right) d_{2} \\
& \leq\left(r_{1}-N_{E}\right) \times\left(r_{2}-N_{E}\right) .
\end{aligned}
$$

When $\min \left(r_{1}, r_{2}\right)>N_{E} \geq \max \left(r_{0}-r_{1}, r_{0}-r_{2}\right)$, the s.d.o.f. region, as illustrated in Fig. 3(c) is bounded by $d_{i} \geq 0$ and the line

$$
\frac{d_{1}}{r_{1}-N_{E}}+\frac{d_{2}}{r_{2}-N_{E}} \leq 1 .
$$

The s.d.o.f. region in Theorem 1 allows the following simple interpretation: the region can be expressed as a convex hull of a set of rectangles shown by Fig. 4 [illustrated for Fig. 3(a)]. Each rectangle is parameterized by the dimensions of the sub-

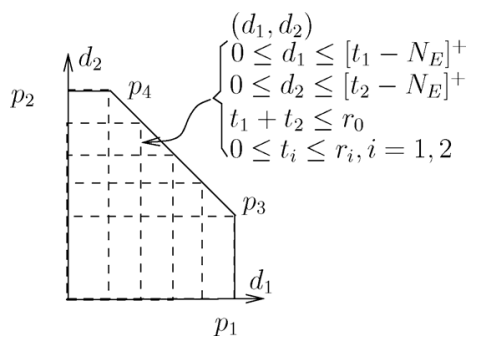

Fig. 4. Interpretation of the s.d.o.f. region as a convex hull of rectangles: $\left(d_{1}, d_{2}\right): 0 \leq d_{i} \leq\left[t_{i}-N_{E}\right]^{+}, i=1,2$, where $t_{i}$ is the number of degrees of freedom occupied by user $i$. To achieve reliable transmission, we must have (20) and (21).

space occupied by the transmission signals from the two users, denoted by $\left(t_{1}, t_{2}\right)$, where $t_{i}$ indicates the dimension of user $i, i=1,2$. Then, in order for the signals from both transmitters to be received reliably by the receiver, we must have

$$
\begin{aligned}
& t_{1}+t_{2} \leq r_{0} \\
& 0 \leq t_{i} \leq r_{i}, i=1,2
\end{aligned}
$$

Each user then transmits confidential messages with $0 \leq d_{i} \leq$ $\left[t_{i}-N_{E}\right]^{+}$over the available $t_{i}$ dimensions, where the $-N_{E}$ term is an effect of the secrecy constraint (4).

It is clear that $p_{3}, p_{4}$ given by (16) and (17) are in one of these rectangles. Hence, the convex hull of these rectangles yields the s.d.o.f. region stated in Theorem 1 .

Finally, we note that if $N_{E} \geq \max \left(N_{T_{1}}, N_{T_{2}}\right)$ the s.d.o.f. region reduces to $(0,0)$ and this implies that secure communication is not possible in this regime.

\section{Proof for the Parallel Channel Model}

In this section, we establish Theorem 1 for the case of parallel channels. As illustrated in Fig. 5, the receiver observes

$$
\begin{aligned}
y_{i}=x_{1 i}+z_{i}, & i \in \mathcal{A}, \\
y_{i}=x_{1 i}+x_{2 i}+z_{i}, & i \in \mathcal{B}, \\
y_{i}=x_{2 i}+z_{i}, & i \in \mathcal{C},
\end{aligned}
$$

where the noise random variables across the subchannels are independent and each is distributed according to $\mathcal{C N}(0,1)$ and $\left\{x_{1 i}\right\}_{i \in \mathcal{A} \cup \mathcal{B}}$ and $\left\{x_{2 i}\right\}_{i \in \mathcal{B} \cup \mathcal{C}}$ denote the transmit symbols of user 1 and user 2 , respectively.

The parallel channel model is a special case of (1) with

$$
\mathbf{H}_{1}=\left[\begin{array}{ccc}
\mathbf{I}_{|\mathcal{A}|} & & \\
& \mathbf{I}_{|\mathcal{B}|} & \\
& & \mathbf{O}_{|\mathcal{C}|}
\end{array}\right], \mathbf{H}_{2}=\left[\begin{array}{lll}
\mathbf{O}_{|\mathcal{A}|} & & \\
& \mathbf{I}_{|\mathcal{B}|} & \\
& & \mathbf{I}_{\mid \mathcal{C}^{\prime}}
\end{array}\right],
$$

where $\mathbf{I}_{|\mathcal{A}|}, \mathbf{I}_{\mid \mathcal{B}}$, and $\mathbf{I}_{\mathcal{C} \mid}$ denote the identity matrices of size $|\mathcal{A}|,|\mathcal{B}|$, and $|\mathcal{C}|$, respectively, and $\mathbf{O}_{|\mathcal{A}|}$ and $\mathbf{O}_{\mathcal{B} \mid}$ denote the matrices, all of whose entries are zeros. Note that we do not make any assumption on the eavesdropper's channel model (2).

\section{A. Achievability}

It suffices to establish the achievability of points $p_{3}$ and $p_{4}$ in (16) and (17), respectively. The rest of the region follows 


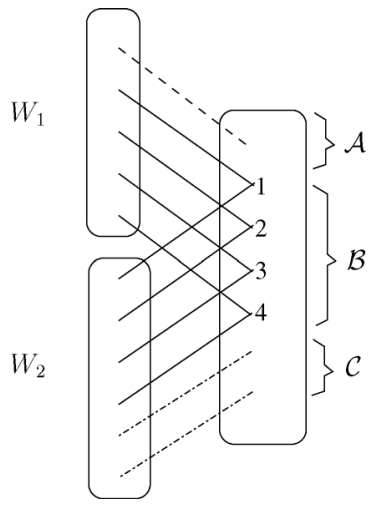

Fig. 5. Definition of the set $\mathcal{A}, \mathcal{B}, \mathcal{C}$, where $|\mathcal{B}|=4$.

through time-sharing between these points. Note that for the proposed parallel channel model

$$
\begin{aligned}
& p_{3}=\left(\left[|\mathcal{A}|+|\mathcal{B}|-N_{E}\right]^{+},\left[|\mathcal{C}|-N_{E}\right]^{+}\right) \\
& p_{4}=\left(\left[|\mathcal{A}|-N_{E}\right]^{+},\left[|\mathcal{B}|+|\mathcal{C}|-N_{E}\right]^{+}\right) .
\end{aligned}
$$

To prove the achievability of $p_{3}$, we restrict user 2 to transmit only on the last $|\mathcal{C}|$ components of (24) and allow user 1 to transmit over all of the components of $\mathcal{A} \cup \mathcal{B}$ in (22) and (23). Note that in this case, the signals of these two users do not interfere with each other at the intended receiver. From [20], user 1 can transmit $W_{1}$ such that $d_{1}=\left[|\mathcal{A}|+|\mathcal{B}|-N_{E}\right]^{+}$and

$$
\lim _{n \rightarrow \infty} \sup _{\tilde{H}_{1}^{n}} I\left(W_{1} ; \tilde{\mathbf{H}}_{1}^{n} \mathbf{X}_{1}^{n} \mid \tilde{\mathbf{H}}_{1}^{n}=\tilde{\mathrm{H}}_{1}^{n}\right)=0
$$

and user 2 can transmit $W_{2}$ such that $d_{2}=\left[|\mathcal{C}|-N_{E}\right]^{+}$and

$$
\lim _{n \rightarrow \infty} \sup _{\tilde{\mathrm{H}}_{2}^{n}} I\left(W_{2} ; \tilde{\mathbf{H}}_{2}^{n} \mathbf{X}_{2}^{n} \mid \tilde{\mathbf{H}}_{2}^{n}=\tilde{\mathrm{H}}_{2}^{n}\right)=0
$$

where we use $\tilde{\mathbf{H}}_{k}^{n} \mathbf{X}_{k}^{n}$ to denote the sequence $\left\{\tilde{\mathbf{H}}_{k}(i) \mathbf{X}_{k}(i), i=\right.$ $1, \ldots, n\}$. Furthermore, since $\left(W_{1}, \mathbf{X}_{1}^{n}\right)$ is independent of $\left(W_{2}, \mathbf{X}_{2}^{n}\right)$ we have that

$$
\begin{aligned}
& \lim _{n \rightarrow \infty} \sup _{\tilde{H}_{1,2}^{n}} I\left(W_{1} ; \tilde{\mathbf{H}}_{1}^{n} \mathbf{X}_{1}^{n}, \tilde{\mathbf{H}}_{2}^{n} \mathbf{X}_{2}^{n} \mid \tilde{\mathbf{H}}_{k}^{n}=\tilde{\mathbf{H}}_{k}^{n},{ }_{k=1,2}\right)=0 \\
& \lim _{n \rightarrow \infty} \sup _{\tilde{H}_{1,2}^{n}} I\left(W_{2} ; \tilde{\mathbf{H}}_{1}^{n} \mathbf{X}_{1}^{n}, \tilde{\mathbf{H}}_{2}^{n} \mathbf{X}_{2}^{n} \mid \tilde{\mathbf{H}}_{k}^{n}=\tilde{\mathbf{H}}_{k}^{n},{ }_{k=1,2}\right)=0 .
\end{aligned}
$$

Note that for $\tilde{\mathbf{H}}_{k}^{n}=\tilde{\mathrm{H}}_{k}^{n}, k=1,2$, we have

$$
\begin{aligned}
& I\left(W_{1} ; \tilde{\mathrm{H}}_{1}^{n} \mathbf{X}_{1}^{n}, \tilde{\mathrm{H}}_{2}^{n} \mathbf{X}_{2}^{n} \mid W_{2}\right) \\
\leq & I\left(W_{1} ; W_{2}, \tilde{\mathrm{H}}_{1}^{n} \mathbf{X}_{1}^{n}, \tilde{\mathrm{H}}_{2}^{n} \mathbf{X}_{2}^{n}\right) \\
= & I\left(W_{1} ; \tilde{\mathrm{H}}_{1}^{n} \mathbf{X}_{1}^{n}, \tilde{\mathrm{H}}_{2}^{n} \mathbf{X}_{2}^{n}\right)+I\left(W_{1} ; W_{2} \mid \tilde{\mathrm{H}}_{1}^{n} \mathbf{X}_{1}^{n}, \tilde{\mathrm{H}}_{2}^{n} \mathbf{X}_{2}^{n}\right) \\
\leq & I\left(W_{1} ; \tilde{\mathrm{H}}_{1}^{n} \mathbf{X}_{1}^{n}, \tilde{\mathrm{H}}_{2}^{n} \mathbf{X}_{2}^{n}\right)+I\left(W_{1}, \tilde{\mathrm{H}}_{1}^{n} \mathbf{X}_{1}^{n} ; W_{2}, \tilde{\mathrm{H}}_{2}^{n} \mathbf{X}_{2}^{n}\right) \\
= & I\left(W_{1} ; \tilde{\mathrm{H}}_{1}^{n} \mathbf{X}_{1}^{n}\right)
\end{aligned}
$$

where the last step follows from the fact that $\left(W_{2}, \tilde{\mathrm{H}}_{2}^{n} \mathbf{X}_{2}^{n}\right)$ is independent from $\left(W_{1}, \tilde{\mathrm{H}}_{1}^{n} \mathbf{X}_{1}^{n}\right)$. Therefore, from (32)-(35) we observe that (28) implies

$$
\lim _{n \rightarrow \infty} \sup _{\tilde{H}_{1,2}^{n}} I\left(W_{1} ; \tilde{\mathbf{H}}_{1}^{n} \mathbf{X}_{1}^{n}, \tilde{\mathbf{H}}_{2}^{n} \mathbf{X}_{2}^{n} \mid W_{2}, \tilde{\mathbf{H}}_{k}^{n}=\tilde{\mathrm{H}}_{k}^{n}, k=1,2\right)=0 .
$$

Using (36) and (31), we obtain

$$
\lim _{n \rightarrow \infty} \sup _{\tilde{\mathrm{H}}_{1,2}^{n}} I\left(W_{1}, W_{2} ; \tilde{\mathbf{H}}_{1}^{n} \mathbf{X}_{1}^{n}, \tilde{\mathbf{H}}_{2}^{n} \mathbf{X}_{2}^{n} \mid \tilde{\mathbf{H}}_{k}^{n}=\tilde{\mathrm{H}}_{k}^{n}, k=1,2\right)=0
$$

and the secrecy constraint (4) follows from the data-processing inequality. Hence, we have proved the point $p_{3}$ is achievable.

The achievability of $p_{4}$ is proved by repeating the argument above by exchanging user 1 with user 2 .

Remark 3: As is evident from (37), the secrecy guarantee achieved by one user is not affected by the transmission strategy of the other user.

\section{B. Converse : $N_{E} \leq \min (|\mathcal{A}|,|\mathcal{C}|)$}

We need to show that the s.d.o.f. region is contained within

$$
\begin{aligned}
d_{1} & \leq|\mathcal{A}|+|\mathcal{B}|-N_{E} \\
d_{2} & \leq|\mathcal{C}|+|\mathcal{B}|-N_{E} \\
d_{1}+d_{2} & \leq|\mathcal{A}|+|\mathcal{B}|+|\mathcal{C}|-2 N_{E} .
\end{aligned}
$$

Since (38) and (39) directly follow from the single user case in [20], we only need to show (40).

Let $\mathcal{E}_{k}$ be the set of links such that an eavesdropper is monitoring for $W_{k}, k=1,2 .\left|\mathcal{E}_{1}\right|=\left|\mathcal{E}_{2}\right|=N_{E} . \mathcal{A} \supseteq \mathcal{E}_{1}, \mathcal{C} \supseteq \mathcal{E}_{2}$. We establish the following upper bound on the achievable rate pairs:

Lemma 1:

$$
\begin{aligned}
& n\left(R_{s, 1}-\delta_{n}\right) \leq I\left(X_{1, \mathcal{A} \backslash \mathcal{E}_{1}}^{n} ; Y_{\mathcal{A} \backslash \mathcal{E}_{1}}^{n}\right)+I\left(X_{1, \mathcal{B}}^{n} ; Y_{\mathcal{B}}^{n} \mid M\right) \\
& n\left(R_{s, 2}-\delta_{n}\right) \leq I\left(X_{2, \mathcal{C} \backslash \mathcal{E}_{2}}^{n} ; Y_{\mathcal{C} \backslash \mathcal{E}_{2}}^{n}\right)+I\left(M ; Y_{\mathcal{B}}^{n}\right)
\end{aligned}
$$

where $M=\left(Y_{\mathcal{A}}^{n}, X_{2, \mathcal{B} \cup \mathcal{C}}^{n}\right)$.

Proof: The proof is provided in Appendix A.

The proof is completed upon adding (41) and (42) so that

$$
\begin{aligned}
& n\left(R_{s, 1}+R_{s, 2}-2 \delta_{n}\right) \\
& \leq\left(X_{1, \mathcal{A} \backslash \mathcal{E}_{1}}^{n} ; Y_{\mathcal{A} \backslash \mathcal{E}_{1}}^{n}\right)+I\left(X_{2, \mathcal{C} \backslash \mathcal{E}_{2}}^{n} ; Y_{\mathcal{C} \backslash \mathcal{E}_{2}}^{n}\right) \\
& \quad+I\left(M, X_{1, \mathcal{B}}^{n} ; Y_{\mathcal{B}}^{n}\right)
\end{aligned}
$$

and using

$$
\begin{aligned}
& d\left(\frac{1}{n} I\left(X_{1, \mathcal{A} \backslash \mathcal{E}_{1}}^{n} ; Y_{\mathcal{A} \backslash \mathcal{E}_{1}}^{n}\right)\right) \leq|\mathcal{A}|-N_{E} \\
& d\left(\frac{1}{n} I\left(X_{2, \mathcal{C} \backslash \mathcal{E}_{2}}^{n} ; Y_{\mathcal{C} \backslash \mathcal{E}_{2}}^{n}\right)\right) \leq|\mathcal{C}|-N_{E} \\
& d\left(\frac{1}{n} I\left(M, X_{1, \mathcal{B}}^{n} ; Y_{\mathcal{B}}^{n}\right)\right) \leq|\mathcal{B}|
\end{aligned}
$$

where $d(x) \triangleq \lim _{P \rightarrow \infty} \frac{x(P)}{\log _{2} P}$ characterizes the prelog scaling of $x$ with respect to $P$. 


\section{Converse : $N_{E}>\max (|\mathcal{A}|,|\mathcal{C}|)$}

Without loss of generality, we assume $|\mathcal{C}| \geq|\mathcal{A}|$. Let $\mathcal{E}_{k}$ be the set of links such that an eavesdropper is monitoring for $W_{k}, k=1,2$. Let $\left|\mathcal{E}_{1}\right|=\left|\mathcal{E}_{2}\right|=N_{E}, \mathcal{A} \subset \mathcal{E}_{1}$, and $\mathcal{C} \subset \mathcal{E}_{2}$.

Define the set $\mathcal{F}, \mathcal{G}$ such that $\mathcal{F}=\mathcal{B} \backslash \mathcal{E}_{1}, \mathcal{G}=\mathcal{B} \backslash \mathcal{E}_{2}$. Since $|\mathcal{C}| \geq|\mathcal{A}|$, we have $|\mathcal{G}| \geq|\mathcal{F}|$.

Then, Theorem 1 reduces to $d_{k} \geq 0, k=1,2$ and

$$
|\mathcal{G}| d_{1}+|\mathcal{F}| d_{2} \leq|\mathcal{F}| \times|\mathcal{G}|
$$

which we show now. We first introduce the following lemma.

Lemma 2: For any choice of $\mathcal{F} \subseteq \mathcal{B}$ and $\mathcal{G} \subseteq \mathcal{B}$ with appropriate cardinalities, the rates $R_{s, 1}$ and $R_{s, 2}$ are upper bounded by

$$
\begin{aligned}
& n\left(R_{s, 1}-\delta_{n}\right) \leq I\left(X_{1, \mathcal{F}}^{n} ; Y_{\mathcal{F}}^{n} \mid M, X_{1, \mathcal{B} \backslash \mathcal{F}}^{n}\right) \\
& n\left(R_{s, 2}-\delta_{n}\right) \leq I\left(M, X_{1, \mathcal{B} \backslash \mathcal{G}}^{n} ; Y_{\mathcal{G}}^{n}\right)
\end{aligned}
$$

where $M=\left\{Y_{\mathcal{A}}^{n}, X_{2 . \mathcal{B} \cup \mathcal{C}}^{n}\right\}$.

Proof: The proof is provided in Appendix B.

For the remainder of the proof, we assume without loss of generality that $\mathcal{B}=\{1, \ldots,|\mathcal{B}|\}$. We fix $\mathcal{G}=\{1, \ldots,|\mathcal{G}|\}$ while choosing $|\mathcal{G}|$ different sets of $|\mathcal{F}|$ elements: $\mathcal{F}_{1}, \ldots, \mathcal{F}_{|\mathcal{G}|}$, the sets $\mathcal{V}_{0}, \ldots, \mathcal{V}_{|\mathcal{G}|}$, and a sequence of $c_{i}$ in the following recursive manner.

Definition 1: Let $\mathcal{V}_{0}=\mathcal{G}, c_{0}=1$. For $i \geq 1$, recursively construct $\mathcal{F}_{i}$ as follows.

1) Case I: $\left|\mathcal{V}_{i-1}\right| \geq|\mathcal{F}|$

Let $\mathcal{F}_{i}=\left\{\mathcal{V}_{i-1}(1), \ldots, \mathcal{V}_{i-1}(|F|)\right\}$, where $\mathcal{V}_{i-1}(k)$ denotes the $k$ th smallest element in $\mathcal{V}_{i-1}$. Let $\mathcal{V}_{i}=\mathcal{V}_{i-1} \backslash \mathcal{F}_{i}$, and $c_{i}=c_{i-1}$. This case is illustrated in Fig. 6(a) for $i=1$.

2) Case II: $\left|\mathcal{V}_{i-1}\right|<\mid \mathcal{F}$

Let $\mathcal{F}_{i}=\mathcal{V}_{i-1} \cup \mathcal{H}_{i}$, and $\mathcal{V}_{i}=\mathcal{G} \backslash \mathcal{H}_{i}$, and $c_{i}=c_{i-1}+1$, where $\mathcal{H}_{i}=\left\{1,2, \ldots,|\mathcal{F}|-\left|\mathcal{V}_{i-1}\right|\right\}$. This case is illustrated in Fig. 6(b) for $i=4$.

To interpret the above construction, we note that the set $\mathcal{G}$ is a row-vector with $|\mathcal{G}|$ elements and let $\mathcal{G}^{\otimes}$ be obtained by concatenating $|\mathcal{F}|$ identical copies of the $\mathcal{G}$ vector, i.e.,

$$
\mathcal{G}^{\otimes}=\underbrace{[\mathcal{G}|\mathcal{G}| \ldots \mathcal{G}]}_{|\mathcal{F}| \text { copies }} .
$$

As shown in Fig. 6 , by our construction, the vector $\mathcal{F}_{1}$ spans the first $|\mathcal{F}|$ elements of $\mathcal{G}^{\otimes}$, the vector $\mathcal{F}_{2}$ spans the next $|\mathcal{F}|$ elements of $\mathcal{G}^{\otimes}$, etc. The constant $c_{i}$ denotes the index number of copies of the $\mathcal{G}$ vector necessary to cover $\mathcal{F}_{i}$.

When $i=|\mathcal{G}|$, the row-vector $\mathcal{F}_{i}$ terminates exactly at the end of the last $\mathcal{G}$ vector in $\mathcal{G}^{\otimes}$. Hence,

$$
c_{|\mathcal{G}|}=|\mathcal{F}|, \quad \mathcal{V}_{|\mathcal{G}|}=\phi
$$

By going through the above recursive procedure and invoking Lemma 2 repeatedly, each time by setting $\mathcal{F}$ in (48) and (49) to

\begin{tabular}{|c|c|c|c|c|c|c|c|c|c|c|c|c|c|}
\hline \multicolumn{2}{|c|}{$\mathcal{F}_{1}$} & \multicolumn{3}{|c|}{$\mathcal{F}_{2}$} & \multicolumn{3}{|c|}{$\mathcal{F}_{3}$} & \multicolumn{3}{|c|}{$\mathcal{F}_{4}$} & & $V_{4}$ \\
\hline 1 & 2 & 3 & 4 & 5 & 6 & 7 & 1 & 2 & 3 & 4 & 5 & & \\
\hline 1 & 2 & 3 & 4 & 5 & 6 & 7 & 1 & 2 & 3 & 4 & 5 & 6 & 7 \\
\hline
\end{tabular}
be $\mathcal{F}_{i}$, we establish the following upper bound on the rate region. (a)

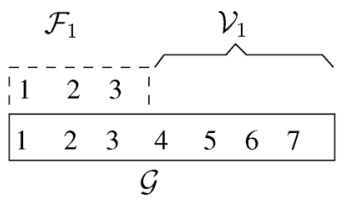

(b)

Fig. 6. The set $\mathcal{F}_{k}, \mathcal{G}$, and $\mathcal{V}_{k}$ when $|\mathcal{F}|=3,|\mathcal{G}|=7$ and $|\mathcal{B}|=8$. (a) Case $\mathrm{I}, i=1, c_{1}=1$. (b) Case II, $i=4, \mathcal{H}_{5}=\{1\}, \mathcal{F}_{5}=\{6,7,1\}, \mathcal{V}_{5}=$ $\{2,3,4,5,6,7\}, c_{4}=2, c_{5}=3$.

Lemma 3: For each $i=0,1, \ldots,|\mathcal{G}|$ and the set of channels $\mathcal{F}_{1}, \mathcal{F}_{2}, \ldots, \mathcal{F}_{|\mathcal{G}|}$ defined in Definition 1 , the rate pair $\left(R_{s, 1}, R_{s, 2}\right)$ satisfies the following upper bound:

$$
\begin{aligned}
i & \cdot n\left(R_{s, 1}-\delta_{n}\right)+c_{i} \cdot n\left(R_{s, 2}-\delta_{n}\right) \\
& \leq \sum_{j=1}^{i} I\left(M, X_{1, \mathcal{B}}^{n} ; Y_{\mathcal{F}_{j}}^{n}\right)+I\left(M, X_{1, \mathcal{B} \backslash \mathcal{G}}^{n} ; Y_{\mathcal{V}_{i}}^{n}\right)
\end{aligned}
$$

Before providing a proof, we note that (47) follows from (52) as described below. Evaluating (52) with $i=|\mathcal{G}|$, using (51) and letting $\tilde{R}_{s, i}=R_{s, i}-\delta_{n}$,

$$
\begin{aligned}
n|\mathcal{G}| \tilde{R}_{s, 1}+n|\mathcal{F}| \tilde{R}_{s, 2} & \leq \sum_{j=1}^{|\mathcal{G}|} I\left(M, X_{1, \mathcal{B}}^{n} ; Y_{\mathcal{F}_{j}}^{n}\right) \\
& =\sum_{j=1}^{|\mathcal{G}|}\left\{h\left(Y_{\mathcal{F}_{j}}^{n}\right)-h\left(Y_{\mathcal{F}_{j}}^{n} \mid M, X_{1, \mathcal{B}}^{n}\right)\right\} \\
& =n\left\{|\mathcal{G}| \cdot|\mathcal{F}| \cdot \log _{2} P+\Theta(1)\right\}
\end{aligned}
$$

where the last step uses the fact that

$$
h\left(Y_{\mathcal{F}_{j}}^{n}\right) \leq \sum_{k \in \mathcal{F}_{j}} h\left(Y_{k}^{n}\right) \leq n\left\{|\mathcal{F}| \log _{2} P+O(1)\right\},
$$

and

$$
h\left(Y_{\mathcal{F}_{j}}^{n} \mid M, X_{1, \mathcal{B}}^{n}\right)=h\left(Y_{\mathcal{F}_{j}}^{n} \mid X_{1, \mathcal{F}_{j}}^{n}, X_{2, \mathcal{F}_{j}}^{n}\right)=n \cdot O(1) .
$$

Dividing each side of (55) by $\log _{2} P$ and taking the limit $P \rightarrow$ $\infty$ yields (47).

Proof of Lemma 3: We use induction over the variable $i$ to establish (52). For $i=0$, note that $c_{0}=0$ and $\mathcal{V}_{1}=\mathcal{G}$ and hence (52) is simply (49). This completes the proof for the base case.

For the induction step, we assume that (52) holds for some $t=i$, we need to show that (52) also holds for $t=i+1$, i.e.,

$$
\begin{aligned}
(i+1) \cdot & n\left(R_{s, 1}-\delta_{n}\right)+c_{i+1} \cdot n\left(R_{s, 2}-\delta_{n}\right) \leq \\
& \sum_{j=1}^{i+1} I\left(M, X_{1, \mathcal{B}}^{n} ; Y_{\mathcal{F}_{j}}^{n}\right)+I\left(M, X_{1, \mathcal{B} \backslash \mathcal{G}}^{n} ; Y_{\mathcal{V}_{i+1}}^{n}\right)
\end{aligned}
$$


holds. For our proof, we separately consider the cases when $|\mathcal{F}| \leq\left|\mathcal{V}_{i}\right|$ and when $\left|\mathcal{V}_{i}\right|<|\mathcal{F}|$ holds.

When $|\mathcal{F}| \leq\left|\mathcal{V}_{i}\right|$, from Definition 1

$$
\mathcal{F}_{i+1} \subseteq \mathcal{V}_{i}, \quad \mathcal{V}_{i+1}=\mathcal{V}_{i} \backslash \mathcal{F}_{i+1}, \quad c_{i+1}=c_{i}
$$

holds. Then, (58) follows by combining (52) with (48) as we show below. Note that

$$
\begin{aligned}
& I\left(M, X_{1, \mathcal{B} \backslash \mathcal{G}}^{n} ; Y_{\mathcal{V}_{i}}^{n}\right)=I\left(M, X_{1, \mathcal{B} \backslash \mathcal{G}}^{n} ; Y_{\mathcal{F}_{i+1}}^{n} \mid Y_{\mathcal{V}_{i} \backslash \mathcal{F}_{i+1}}^{n}\right) \\
& \quad+I\left(M, X_{1, \mathcal{B} \backslash \mathcal{G}}^{n} ; Y_{\mathcal{V}_{i+1}}^{n}\right) \\
& \leq I\left(M, X_{1, \mathcal{B} \backslash \mathcal{G}}^{n}, Y_{\mathcal{V}_{i} \backslash \mathcal{F}_{i+1}}^{n} ; Y_{\mathcal{F}_{i+1}}^{n}\right)+I\left(M, X_{1, \mathcal{B} \backslash \mathcal{G}}^{n} ; Y_{\mathcal{V}_{i+1}}^{n}\right) \\
& \leq I\left(M, X_{1, \mathcal{B} \backslash \mathcal{G}}^{n}, X_{1, \mathcal{V}_{i} \backslash \mathcal{F}_{i+1}}^{n} ; Y_{\mathcal{F}_{i+1}}^{n}\right)+I\left(M, X_{1, \mathcal{B} \backslash \mathcal{G}}^{n} ; Y_{\mathcal{V}_{i+1}}^{n}\right) \\
& \leq I\left(M, X_{1, \mathcal{B} \backslash \mathcal{G}}^{n}, X_{1, \mathcal{G} \backslash \mathcal{F}_{i+1}}^{n} ; Y_{\mathcal{F}_{i+1}}^{n}\right)+I\left(M, X_{1, \mathcal{B} \backslash \mathcal{G}}^{n} ; Y_{\mathcal{V}_{i+1}}^{n}\right) \\
& =I\left(M, X_{1, \mathcal{B} \backslash \mathcal{F}_{i+1}}^{n} ; Y_{\mathcal{F}_{i+1}}^{n}\right)+I\left(M, X_{1, \mathcal{B} \backslash \mathcal{G}}^{n} ; Y_{\mathcal{V}_{i+1}}^{n}\right)
\end{aligned}
$$

where (60) follows from the chain rule of the mutual information and the definition of $\mathcal{V}_{i+1}$ in (59), while (62) follows from the Markov condition

$Y_{\mathcal{V}_{i} \backslash \mathcal{F}_{i+1}}^{n} \leftrightarrow\left(X_{1, \mathcal{V}_{i} \backslash \mathcal{F}_{i+1}}^{n}, X_{2, \mathcal{V}_{i} \backslash \mathcal{F}_{i+1}}^{n}\right) \leftrightarrow\left(M, Y_{\mathcal{F}_{i+1}}^{n}, X_{1, \mathcal{B} \backslash \mathcal{G}}^{n}\right)$

and the fact that $M=\left(X_{2, \mathcal{B} \cup \mathcal{C}}^{n}, Y_{\mathcal{A}}^{n}\right)$ already includes $X_{2, \mathcal{V}_{i} \backslash \mathcal{F}_{i+1}},(63)$ follows from the fact that $\mathcal{V}_{i} \subseteq \mathcal{G}$, while (64) follows from the fact that $\{\mathcal{B} \backslash \mathcal{G}\} \cup\left\{\mathcal{G} \backslash \mathcal{F}_{i+1}\right\}=\left\{\mathcal{B} \backslash \mathcal{F}_{i+1}\right\}$.

Substituting (64) into the last term in (52), we obtain

$$
\begin{aligned}
& i \cdot n\left(R_{s, 1}-\delta_{n}\right)+c_{i} \cdot n\left(R_{s, 2}-\delta_{n}\right) \\
& \leq \sum_{j=1}^{i} I\left(M, X_{1, \mathcal{B}}^{n} ; Y_{\mathcal{F}_{j}}^{n}\right)+I\left(M, X_{1, \mathcal{B} \backslash \mathcal{G}}^{n} ; Y_{\mathcal{V}_{i}}^{n}\right) \\
& \leq \sum_{j=1}^{i} I\left(M, X_{1, \mathcal{B}}^{n} ; Y_{\mathcal{F}_{j}}^{n}\right)+I\left(M, X_{1, \mathcal{B} \backslash \mathcal{F}_{i+1}}^{n} ; Y_{\mathcal{F}_{i+1}}^{n}\right) \\
& \quad+I\left(M, X_{1, \mathcal{B} \backslash \mathcal{G}}^{n} ; Y_{\mathcal{V}_{i+1}}^{n}\right) .
\end{aligned}
$$

Finally, combining (66) with (48) and using $c_{i+1}=c_{i}$ [cf. (59)] we have

$$
\begin{aligned}
& (i+1) \cdot n\left(R_{s, 1}-\delta_{n}\right)+c_{i+1} \cdot n\left(R_{s, 2}-\delta_{n}\right) \\
& \leq \sum_{j=1}^{i} I\left(M, X_{1, \mathcal{B}}^{n} ; Y_{\mathcal{F}_{j}}^{n}\right)+I\left(M, X_{1, \mathcal{B} \backslash \mathcal{F}_{i+1}}^{n} ; Y_{\mathcal{F}_{i+1}}^{n}\right) \\
& \quad+I\left(M, X_{1, \mathcal{B} \backslash \mathcal{G}}^{n} ; Y_{\mathcal{V}_{i+1}}^{n}\right) \\
& \quad \quad+I\left(X_{1, \mathcal{F}_{i+1}}^{n} ; Y_{\mathcal{F}_{i+1}}^{n} \mid M, X_{1, \mathcal{B} \backslash \mathcal{F}_{i+1}}^{n}\right) \\
& \quad \sum_{j=1}^{i+1} I\left(M, X_{1, \mathcal{B}}^{n} ; Y_{\mathcal{F}_{j}}^{n}\right)+I\left(M, X_{1, \mathcal{B} \backslash \mathcal{G}}^{n} ; Y_{\mathcal{V}_{i+1}}^{n}\right)
\end{aligned}
$$

as required.
When $|\mathcal{F}|>\left|\mathcal{V}_{i}\right|$, as stated in Definition 1 we introduce $\mathcal{H}_{i+1}=\left\{1,2, \ldots,|\mathcal{F}|-\left|\mathcal{V}_{i}\right|\right\}$ and recall that

$\mathcal{F}_{i+1}=\mathcal{V}_{i} \cup \mathcal{H}_{i+1}, \quad \mathcal{V}_{i+1}=\mathcal{G} \backslash \mathcal{H}_{i+1}, \quad c_{i+1}=c_{i}+1$

holds. From (49) and (58), we have that

$$
\begin{aligned}
& i \cdot n\left(R_{s, 1}-\delta_{n}\right)+\left(c_{i}+1\right) \cdot n\left(R_{s, 2}-\delta_{n}\right) \\
& =\sum_{j=1}^{i} I\left(M, X_{1, \mathcal{B}}^{n} ; Y_{\mathcal{F}_{j}}^{n}\right)+I\left(M, X_{1, \mathcal{B} \backslash \mathcal{G}}^{n} ; Y_{\mathcal{V}_{i}}^{n}\right) \\
& \quad+I\left(M, X_{1, \mathcal{B} \backslash \mathcal{G}}^{n} ; Y_{\mathcal{G}}^{n}\right) \\
& \quad \sum_{j=1}^{i} I\left(M, X_{1, \mathcal{B}}^{n} ; Y_{\mathcal{F}_{j}}^{n}\right)+I\left(M, X_{1, \mathcal{B} \backslash \mathcal{G}}^{n} ; Y_{\mathcal{V}_{i}}^{n}\right) \\
& \quad+I\left(M, X_{1, \mathcal{B} \backslash \mathcal{G}}^{n} ; Y_{\mathcal{H}_{i+1}}^{n} \mid Y_{\mathcal{G} \backslash \mathcal{H}_{i+1}}^{n}\right)+I\left(M, X_{1, \mathcal{B} \backslash \mathcal{G}}^{n} ; Y_{\mathcal{V}_{i+1}}^{n}\right) .
\end{aligned}
$$

As we will show subsequently,

$$
\begin{array}{r}
I\left(M, X_{1, \mathcal{B} \backslash \mathcal{G}}^{n} ; Y_{\mathcal{V}_{i}}^{n}\right)+I\left(M, X_{1, \mathcal{B} \backslash \mathcal{G}}^{n} ; Y_{\mathcal{H}_{i+1}}^{n} \mid Y_{\mathcal{G} \backslash \mathcal{H}_{i+1}}^{n}\right) \\
\leq I\left(M, X_{1, \mathcal{B} \backslash \mathcal{F}_{i+1}}^{n} ; Y_{\mathcal{F}_{i+1}}^{n}\right)
\end{array}
$$

Combining (48), (71), and (72) and using $c_{i+1}=c_{i}+1$ we obtain that

$$
\begin{aligned}
& (i+1) \cdot n\left(R_{s, 1}-\delta_{n}\right)+c_{i+1} \cdot n\left(R_{s, 2}-\delta_{n}\right) \\
& \leq \sum_{j=1}^{i} I\left(M, X_{1, \mathcal{B}}^{n} ; Y_{\mathcal{F}_{j}}^{n}\right)+I\left(M, X_{1, \mathcal{B} \backslash \mathcal{G}}^{n} ; Y_{\mathcal{V}_{i+1}}^{n}\right) \\
& \quad+I\left(M, X_{1, \mathcal{B} \backslash \mathcal{F}_{i+1}}^{n} ; Y_{\mathcal{F}_{i+1}}^{n}\right)+I\left(X_{\mathcal{F}_{i+1}}^{n} ; Y_{\mathcal{F}_{i+1}}^{n} \mid M, X_{1, \mathcal{B} \backslash \mathcal{F}_{i+1}}^{n}\right) \\
& =\sum_{j=1}^{i} I\left(M, X_{1, \mathcal{B}}^{n} ; Y_{\mathcal{F}_{j}}^{n}\right)+I\left(M, X_{1, \mathcal{B} \backslash \mathcal{G}}^{n} ; Y_{\mathcal{V}_{i+1}}^{n}\right) \\
& \quad+I\left(M, X_{1, \mathcal{B}}^{n} ; Y_{\mathcal{F}_{i+1}}^{n}\right),
\end{aligned}
$$

which establishes (58).

It only remains to establish (72) which we do now. First, since $\mathcal{F}_{i+1} \subseteq \mathcal{G}$ it follows that $\{\mathcal{B} \backslash \mathcal{G}\} \subseteq\left\{\mathcal{B} \backslash \mathcal{F}_{i+1}\right\}$ and hence we bound the first term in the left-hand side of (72) as

$$
I\left(M, X_{1, \mathcal{B} \backslash \mathcal{G}}^{n} ; Y_{\mathcal{V}_{i}}^{n}\right) \leq I\left(M, X_{1, \mathcal{B} \backslash \mathcal{F}_{i+1}}^{n} ; Y_{\mathcal{V}_{i}}^{n}\right) .
$$

Next, since the set $\mathcal{H}_{i+1}=\left\{1, \ldots,|\mathcal{F}|-\left|\mathcal{V}_{i}\right|\right\}$ constitutes the first $|\mathcal{F}|-\left|\mathcal{V}_{i}\right|$ elements of $\mathcal{G}$ and $\mathcal{V}_{i}=\left\{|\mathcal{G}|-\left|\mathcal{V}_{i}\right|+1, \ldots,|\mathcal{G}|\right\}$ constitutes the last $\left|\mathcal{V}_{i}\right|$ elements of $\mathcal{G}$ and $|\mathcal{F}| \leq|\mathcal{G}|$ we have that

$$
\begin{aligned}
& \left\{\mathcal{G} \backslash \mathcal{H}_{i+1}\right\}=\left\{|\mathcal{F}|-\left|\mathcal{V}_{i}\right|+1, \ldots,|\mathcal{G}|\right\} \\
& =\left\{|\mathcal{F}|-\left|\mathcal{V}_{i}\right|+1, \ldots,|\mathcal{G}|-\left|\mathcal{V}_{i}\right|\right\} \cup\left\{|\mathcal{G}|-\left|\mathcal{V}_{i}\right|+1, \ldots,|\mathcal{G}|\right\} \\
& =\left\{\mathcal{G} \backslash\left(\mathcal{H}_{i+1} \cup \mathcal{V}_{i}\right)\right\} \cup \mathcal{V}_{i} \\
& =\left\{\mathcal{G} \backslash \mathcal{F}_{i+1}\right\} \cup \mathcal{V}_{i}
\end{aligned}
$$


where the last relation follows from the definition of $\mathcal{F}_{i+1}$ [cf. (69)]. Using (76), we can bound the second term in (72) as follows:

$$
\begin{aligned}
& I\left(M, X_{1, \mathcal{B} \backslash \mathcal{G}}^{n} ; Y_{\mathcal{H}_{i+1}}^{n} \mid Y_{\mathcal{G} \backslash \mathcal{H}_{i+1}}^{n}\right) \\
& \quad=I\left(M, X_{1, \mathcal{B} \backslash \mathcal{G}}^{n} ; Y_{\mathcal{H}_{i+1}}^{n} \mid Y_{\mathcal{G} \backslash \mathcal{F}_{i+1}}^{n}, Y_{\mathcal{V}_{i}}^{n}\right) \\
& \leq I\left(M, X_{1, \mathcal{B} \backslash \mathcal{G}}^{n}, Y_{G \backslash \mathcal{F}_{i+1}}^{n} ; Y_{\mathcal{H}_{i+1}}^{n} \mid Y_{\mathcal{V}_{i}}^{n}\right) \\
& \leq I\left(M, X_{1, \mathcal{B} \backslash \mathcal{G}}^{n}, X_{1, \mathcal{G} \backslash \mathcal{F}_{i+1}}^{n} ; Y_{\mathcal{H}_{i+1}}^{n} \mid Y_{\mathcal{V}_{i}}^{n}\right) \\
& \leq I\left(M, X_{1, \mathcal{B} \backslash \mathcal{F}_{i+1}}^{n} ; Y_{\mathcal{H}_{i+1}}^{n} \mid Y_{\mathcal{V}_{i}}^{n}\right),
\end{aligned}
$$

where in (79), we use the Markov relation

$$
Y_{\mathcal{G} \backslash \mathcal{F}_{i+1}}^{n} \leftrightarrow\left(X_{1, \mathcal{G} \backslash \mathcal{F}_{i+1}}^{n}, X_{2, \mathcal{G} \backslash \mathcal{F}_{i+1}}^{n}\right) \leftrightarrow\left(M, X_{1, \mathcal{B} \backslash \mathcal{G}}^{n}, Y_{\mathcal{F}_{i+1}}^{n}\right)
$$

and the fact that $M=\left(X_{2, \mathcal{B} \cup \mathcal{C}}^{n}, Y_{\mathcal{A}}^{n}\right)$ already contains $X_{2, \mathcal{G} \backslash \mathcal{F}_{i+1}}^{n}$. Combining (75) and (80) gives

$$
\begin{aligned}
I\left(M, X_{1, \mathcal{B} \backslash \mathcal{G}}^{n} ; Y_{\mathcal{V}_{i}}^{n}\right)+I & \left(M, X_{1, \mathcal{B} \backslash \mathcal{G}}^{n} ; Y_{\mathcal{H}_{i+1}}^{n} \mid Y_{\mathcal{G} \backslash \mathcal{H}_{i+1}}^{n}\right) \\
& \leq I\left(M, X_{1, \mathcal{B} \backslash \mathcal{F}_{i+1}}^{n} ; Y_{\mathcal{F}_{i+1}}^{n}\right),
\end{aligned}
$$

thus establishing (72).

This completes the proof.

\section{Converse: $\min (|\mathcal{A}|,|\mathcal{C}|) \leq N_{E} \leq \max (|\mathcal{A}|,|\mathcal{C}|)$}

We assume without loss of generality that $|\mathcal{C}| \geq|\mathcal{A}|$ and as before let $\mathcal{E}_{k}$ be the set of links such that an eavesdropper is monitoring for message $W_{k}$. Since $\left|\mathcal{E}_{1}\right|=\left|\mathcal{E}_{2}\right|=N_{E}$ and $|\mathcal{A}| \leq N_{E} \leq|\mathcal{C}|$ holds, we select the sets such that the relations $\mathcal{A} \subseteq \mathcal{E}_{1} \subseteq \mathcal{A} \cup \mathcal{B}$ and $\mathcal{C} \supseteq \mathcal{E}_{2}$ are both satisfied. Define $\mathcal{F}=$ $\mathcal{B} \backslash \overline{\mathcal{E}}_{1}$ and note that $|\mathcal{F}|=|\mathcal{A}|+|\mathcal{B}|-N_{E}$.

Theorem 1 reduces to the following region:

$$
\begin{aligned}
& 0 \leq d_{1} \leq|\mathcal{F}| \\
& 0 \leq d_{2} \leq|\mathcal{B}|+|\mathcal{C}|-N_{E} \\
& |\mathcal{B}| d_{1}+|\mathcal{F}| d_{2} \leq\left(|\mathcal{B}|+|\mathcal{C}|-N_{E}\right) \times|F|
\end{aligned}
$$

Since (83) and (84) directly follow from the single user case [20], we only need to establish (85). As in earlier cases we begin by establishing the following bounds on the rate pair $\left(R_{s, 1}, R_{s, 2}\right)$ :

$$
\begin{aligned}
& n\left(R_{s, 1}-\delta_{n}\right) \leq I\left(X_{1, \mathcal{F}}^{n} ; Y_{\mathcal{F}}^{n} \mid M, X_{1, \mathcal{B} \backslash \mathcal{F}}^{n}\right) \\
& n\left(R_{s, 2}-\delta_{n}\right) \leq I\left(M ; Y_{\mathcal{B}}^{n}\right)+I\left(X_{2, \mathcal{C} \backslash \mathcal{E}_{2}}^{n} ; Y_{\mathcal{C} \backslash \mathcal{E}_{2}}^{n}\right)
\end{aligned}
$$

where $M=\left(X_{2, \mathcal{B} \cup \mathcal{C}}^{n}, Y_{\mathcal{A}}^{n}\right)$.

Proof: The proof for (86) is identical to (48) in Lemma 2 since the proof does not depend on the choice of $\mathcal{E}_{2}$. The proof for (87) is identical to (42) in Lemma 1.

To establish (83)-(85), note that by defining

$$
R_{s, 2}^{\prime}=R_{s, 2}-\frac{1}{n} I\left(X_{2, \mathcal{C} \backslash \mathcal{E}_{2}}^{n} ; Y_{\mathcal{C} \backslash \mathcal{E}_{2}}^{n}\right),
$$

we have from (87) that

$$
n\left(R_{s, 2}^{\prime}-\delta_{n}\right) \leq I\left(M ; Y_{\mathcal{B}}^{n}\right)
$$

and the bounds on $R_{s, 1}$ and $R_{s, 2}^{\prime}$ in (86) and (89) are identical to the bounds (48) and (49) in Lemma 2 with $\mathcal{G}=\mathcal{B}$. Applying
Lemma 3 to $R_{s, 1}$ and $R_{s, 2}^{\prime}$ for each $i=0,1, \cdots,|\mathcal{G}|$, it follows that

$$
\begin{aligned}
i & \cdot n\left(R_{s, 1}-\delta_{n}\right)+c_{i} \cdot n\left(R_{s, 2}^{\prime}-\delta_{n}\right) \\
\leq & \sum_{j=1}^{i} I\left(M, X_{1, \mathcal{B}}^{n} ; Y_{\mathcal{F}_{j}}^{n}\right)+I\left(M, Y_{\mathcal{V}_{i}}^{n}\right),
\end{aligned}
$$

where the sets $\mathcal{V}_{i}, \mathcal{F}_{i}$ and the sequence $c_{i}$ are as in Definition 1. Substituting (89) into (90) and evaluating the bound for $i=|\mathcal{B}|$, we have that

$$
\begin{array}{r}
|\mathcal{B}| n\left(R_{s, 1}-\delta_{n}\right)+|\mathcal{F}| n\left(R_{s, 2}-\delta_{n}\right) \leq|\mathcal{F}| I\left(X_{2, \mathcal{C} \backslash \mathcal{E}_{2}}^{n} ; Y_{\mathcal{C} \backslash \mathcal{E}_{2}}^{n}\right) \\
+\sum_{j=1}^{|\mathcal{B}|} I\left(M, X_{1, \mathcal{B}}^{n} ; Y_{\mathcal{F}_{j}}^{n}\right)
\end{array}
$$

Finally, substituting

$$
\begin{aligned}
& d\left(\frac{1}{n} I\left(M, X_{2, \mathcal{C} \backslash \mathcal{E}_{2}}^{n} ; Y_{\mathcal{C} \backslash \mathcal{E}_{2}}^{n}\right) \leq|\mathcal{C}|-N_{E}\right. \\
& d\left(\frac{1}{n} I\left(M, X_{1, \mathcal{B}}^{n} ; Y_{\mathcal{F}_{j}}^{n}\right)\right) \leq|\mathcal{F}|,
\end{aligned}
$$

in (91) we obtain (85).

\section{General MIMO-MAC}

The result for the general MIMO case (1) follows by a transformation that reduces the model to the case of parallel independent channels in the previous section while preserving the s.d.o.f. region. As we discuss next, this transformation involves the GSVD [35] and a channel enhancement argument. For an analogous application of GSVD to broadcast channels, see e.g., [21], [36], [37]. We note that channel enhancement techniques are used in many different problems in multiuser information theory; see e.g., [12].

\section{A. GSVD Transformation}

Theorem 2 [35]: Given a pair of matrices $\mathbf{H}_{1}$ and $\mathbf{H}_{2}$ such that the rank of $\mathbf{H}_{i}$ is $r_{i}, i=1,2$, and the rank of $\left[\mathbf{H}_{1} \mid \mathbf{H}_{2}\right]$ is $r_{0}$, there exists unitary matrices $\mathbf{U}_{1}, \mathbf{U}_{2}, \mathbf{W}, \mathbf{Q}$ and nonsingular upper triangular matrix $\mathbf{R}$ such that for $s=r_{1}+r_{2}-r_{0}, \tilde{r}_{1}=$ $r_{1}-s, \tilde{r}_{2}=r_{2}-s$,

$$
\begin{aligned}
& \mathbf{U}_{1}^{H} \mathbf{H}_{1}^{H} \mathbf{Q}=\boldsymbol{\Sigma}_{1\left(N_{T_{1}} \times r_{0}\right)}\left[\mathbf{W}^{H} \mathbf{R}_{\left(r_{0} \times r_{0}\right)}, \mathbf{0}\right]_{\left(r_{0} \times N_{R}\right)} \\
& \mathbf{U}_{2}^{H} \mathbf{H}_{2}^{H} \mathbf{Q}=\boldsymbol{\Sigma}_{2\left(N_{T_{2}} \times r_{0}\right)}\left[\mathbf{W}^{H} \mathbf{R}_{\left(r_{0} \times r_{0}\right)}, \mathbf{0}\right]_{\left(r_{0} \times N_{R}\right)} \\
& \boldsymbol{\Sigma}_{1}=\left[\begin{array}{lll}
\mathbf{I}_{1\left(\tilde{r}_{1} \times \tilde{r}_{1}\right)} & \\
& \mathbf{S}_{1(s \times s)} & \\
& \mathbf{O}_{1\left(\left(N_{T_{1}}-\tilde{r}_{1}-s\right) \times \tilde{r}_{2}\right)}
\end{array}\right] \\
& \boldsymbol{\Sigma}_{2}=\left[\begin{array}{lll}
\mathbf{O}_{2\left(\left(N_{T_{2}}-\tilde{r}_{2}-s\right) \times \tilde{r}_{1}\right)} & \mathbf{S}_{2(s \times s)} \\
& & \mathbf{I}_{2\left(\tilde{r}_{2} \times \tilde{r}_{2}\right)}
\end{array}\right]
\end{aligned}
$$

where $\mathbf{I}_{i}, i=1,2$ are $\tilde{r}_{i} \times \tilde{r}_{i}$ identity matrices, $\mathbf{O}_{i}, i=1,2$ are zero matrices, and $\mathbf{S}_{i}, i=1,2$ are $s \times s$ diagonal matrices with positive real elements on the diagonal line that satisfy $\mathbf{S}_{1}^{2}+\mathbf{S}_{2}^{2}=$ $\mathbf{I}_{s}$, and $\tilde{r}_{1}+s+\tilde{r}_{2}=r_{0}$. For clarity, the dimension of each matrix is shown in the parenthesis in the subscript. $\mathbf{I}_{1}$ has the same number of columns as $\mathbf{O}_{2} . \mathbf{I}_{2}$ has the same number of 
columns $\mathbf{O}_{1}$. However, $\mathbf{O}_{i}, i=1,2$ are not necessarily square matrices and can be empty, i.e., having zero number of rows.

For convenience in notation, we define $\mathbf{A}=\mathbf{W}^{H} \mathbf{R}$ and observe that $\mathbf{A}$ is a square and nonsingular matrix. Then from Theorem 2, we have

$$
\mathbf{Q}^{H} \mathbf{H}_{t} \mathbf{U}_{t}=\left[\begin{array}{l}
\mathbf{A}^{H} \\
\mathbf{0}
\end{array}\right] \boldsymbol{\Sigma}_{t}^{H}, t=1,2 .
$$

Without loss of generality, we can cancel $\mathbf{Q}$ and $\mathbf{U}_{t}$ and rewrite (1) as

$$
\begin{aligned}
\mathbf{Y}=\left[\begin{array}{l}
\mathbf{A}_{r_{0} \times r_{0}}^{H} \\
\mathbf{0}_{\left(N_{R}-r_{0}\right) \times r_{0}}^{H}
\end{array}\right]_{N_{R} \times r_{0}} \boldsymbol{\Sigma}_{1}^{H} \mathbf{X}_{1} \\
\\
\quad+\left[\begin{array}{l}
\mathbf{A}_{r_{0} \times r_{0}}^{H} \\
\mathbf{0}_{\left(N_{R}-r_{0}\right) \times r_{0}}
\end{array}\right]_{N_{R} \times r_{0}} \boldsymbol{\Sigma}_{2}^{H} \mathbf{X}_{2}+\mathbf{Z} .
\end{aligned}
$$

Since $\mathbf{Q}$ and $\mathbf{U}_{t}$ are unitary matrices, the components of $\mathbf{Z}$ are independent from each other and the power constraints of each transmitter remains the same as $\bar{P}_{i}, i=1,2$. Because the components of $\mathbf{Z}$ are independent, the intended receiver can discard the last $N_{R}-r_{0}$ components in $\mathbf{Y}$ without affecting the secrecy capacity region of this channel. This means that we only need to consider the case where $N_{R}=r_{0}$ and rewrite (1) as

$$
\mathbf{Y}=\mathbf{A}_{r_{0} \times r_{0}}^{H}\left(\boldsymbol{\Sigma}_{1}^{H} \mathbf{X}_{1}+\boldsymbol{\Sigma}_{2}^{H} \mathbf{X}_{2}\right)+\mathbf{Z}
$$

\section{B. Converse}

For establishing the converse, we further enhance the channel model in (100) to the following:

$$
\mathbf{Y}=\boldsymbol{\Sigma}_{1}^{H} \mathbf{X}_{1}+\boldsymbol{\Sigma}_{2}^{H} \mathbf{X}_{2}+\sigma_{+} \mathbf{Z}^{\prime}
$$

where $\sigma_{+} \leq 1$ is any sufficiently small constant such that, $\sigma_{+}^{2}$ times the maximal eigenvalue of $\mathbf{A}_{r_{0} \times r_{0}}^{H} \mathbf{A}_{r_{0} \times r_{0}}$, is smaller than 1 and $\mathbf{Z}^{\prime}$ is a circularly symmetric unit-variance Gaussian noise vector.

To establish (101), note that we can express

$$
\mathbf{Z}=\sigma_{+} \cdot \mathbf{A}^{H} \mathbf{Z}^{\prime}+\mathbf{Z}^{\prime}
$$

where $\mathbf{Z}^{\prime \prime}$ is a Gaussian random vector, independent of $\mathbf{Z}^{\prime}$ and with a covariance matrix

$$
\mathbf{I}_{r_{0} \times r_{0}}-\sigma_{+}^{2} \mathbf{A}_{r_{0} \times r_{0}}^{H} \mathbf{A}_{r_{0} \times r_{0}}
$$

which is guaranteed to be positive semidefinite by our choice of $\sigma_{+}$. Upon substituting (102) into (100), we have

$$
\mathbf{Y}=\mathbf{A}_{r_{0} \times r_{0}}^{H}\left(\boldsymbol{\Sigma}_{1}^{H} \mathbf{X}_{1}+\boldsymbol{\Sigma}_{2}^{H} \mathbf{X}_{2}+\sigma_{+} \mathbf{Z}^{\prime}\right)+\mathbf{Z}^{\prime \prime} .
$$

We consider an enhanced receiver that is revealed $\mathbf{Z}^{\prime \prime}$. Clearly, this additional knowledge can only increase the rate and serves as an upper bound. It is also clear that since $\mathbf{Z}^{\prime \prime}$ is independent of $\left(\mathbf{X}_{1}, \mathbf{X}_{2}, \mathbf{Z}^{\prime}\right)$, it suffices to use this information to cancel $\mathbf{Z}^{\prime \prime}$ in (104) and then discard it. Furthermore, since the matrix $\mathbf{A}$ is invertible, upon canceling it, we obtain (101).
We further enhance the receiver by replacing $\boldsymbol{\Sigma}_{1}^{H}$ and $\boldsymbol{\Sigma}_{2}^{H}$ with $\overline{\boldsymbol{\Sigma}}_{1}^{H}$ and $\overline{\mathbf{\Sigma}}_{2}^{H}$ so that the model reduces to

$$
\mathbf{Y}=\overline{\bar{\Sigma}}_{1}^{H} \mathbf{X}_{1}+\overline{\mathbf{\Sigma}}_{2}^{H} \mathbf{X}_{2}+\sigma_{+} \mathbf{Z}^{\prime}
$$

where

$$
\begin{aligned}
& \overline{\boldsymbol{\Sigma}}_{1}^{H}=\left[\begin{array}{lll}
\mathbf{I}_{\tilde{r}_{1} \times \tilde{r}_{1}} & & \\
& \mathbf{I}_{1(s \times s)} & \\
& & \mathbf{0}_{1\left(\tilde{r}_{2} \times\left(N_{T_{1}}-r_{1}\right)\right)}
\end{array}\right]_{r_{0} \times N_{T_{1}}} \\
& \overline{\boldsymbol{\Sigma}}_{2}^{H}=\left[\begin{array}{lll}
\mathbf{0}_{2\left(\tilde{r}_{1} \times\left(N_{T_{2}}-r_{2}\right)\right)} & \\
& \mathbf{I}_{2(s \times s)} & \\
& & \mathbf{I}_{2\left(\tilde{r}_{2} \times \tilde{r}_{2}\right)}
\end{array}\right]_{r_{0} \times N_{T_{2}}}
\end{aligned}
$$

are obtained by replacing each diagonal $\mathbf{S}_{i}$ by the identity matrix. The model (105) can only have a higher capacity, since each diagonal entry in $\mathbf{S}_{i}$ is between $(0,1)$. We observe that in the resulting channel, model is identical to (22)-(24)

$$
\begin{aligned}
& |\mathcal{A}|=r_{0}-r_{2} \\
& |\mathcal{B}|=s=r_{1}+r_{2}-r_{0} \\
& |\mathcal{C}|=r_{0}-r_{1}
\end{aligned}
$$

except that the noise variance is reduced by a factor of $\sigma_{+}^{2}$. Since a fixed scaling in the noise power does not affect the securedegrees of freedom, an outer bound on the s.d.o.f. for the parallel channel model (22)-(24) with $\mathcal{A}, \mathcal{B}$, and $\mathcal{C}$ defined via (105), continues to be an outer bound on the s.d.o.f. region for the general MIMO-MAC channel.

Substituting (108)-(110) in the upper bounds in Sections V-B, $\mathrm{V}-\mathrm{C}$, and V-D, we establish the converse in Theorem 1.

\section{Achievability}

To establish the achievability for the general MIMO case, we further use a suitable degradation mechanism to reduce the model (100) to

$$
\mathbf{Y}=\boldsymbol{\Sigma}_{1}^{H} \mathbf{X}_{1}+\boldsymbol{\Sigma}_{2}^{H} \mathbf{X}_{2}+\sigma \mathbf{Z}^{\prime \prime}
$$

where $\sigma \geq 1$ is any sufficiently large constant such that, $\sigma^{2}$ times the minimum eigenvalue of $\mathbf{A}_{r_{0} \times r_{0}}^{H} \mathbf{A}_{r_{0} \times r_{0}}$, is greater than 1 and $\mathbf{Z}^{\prime \prime}$ is a circularly symmetric unit-variance Gaussian noise vector. Since $\mathbf{A}$ is nonsingular, we are guaranteed that all the singular values of $\mathbf{A}$ are nonzero and hence a $\sigma<\infty$ exists.

To establish (111), let $\mathbf{Z}^{\prime}$ be a Gaussian noise vector with covariance

$$
\sigma^{2} \mathbf{A}_{r_{0} \times r_{0}}^{H} \mathbf{A}_{r_{0} \times r_{0}}-\mathbf{I}_{r_{0} \times r_{0}}
$$

independent of $\mathbf{Z}$ and consider a degraded version of (100)

$$
\mathbf{Y}=\mathbf{A}_{r_{0} \times r_{0}}^{H}\left(\boldsymbol{\Sigma}_{1}^{H} \mathbf{X}_{1}+\boldsymbol{\Sigma}_{2}^{H} \mathbf{X}_{2}\right)+\mathbf{Z}+\mathbf{Z}^{\prime}
$$

which can be simulated at the receiver by adding additional noise $\mathbf{Z}^{\prime}$ to its output. Since $\mathbf{Z}+\mathbf{Z}^{\prime} \sim \mathcal{C N}\left(0, \sigma^{2} \mathbf{A}^{H} \mathbf{A}\right)$, we can express $\mathbf{Z}+\mathbf{Z}^{\prime}=\sigma \mathbf{A}^{H} \mathbf{Z}^{\prime \prime}$. Substituting into (113) and canceling the nonsingular matrix $\mathbf{A}$, we arrive at (111). 
Let $\bar{s}>0$ denote the minimum element on the diagonals of $\mathbf{S}_{1}$ and $\mathbf{S}_{2}$ in (96) and (97), respectively. By appropriately scaling down the transmit powers on each of the subchannels, we can further reduce (104) to

$$
\mathbf{Y}=\overline{\bar{\Sigma}}_{1}^{H} \mathbf{X}_{1}+\overline{\mathbf{\Sigma}}_{2}^{H} \mathbf{X}_{2}+\frac{\sigma}{\bar{s}} \mathbf{Z}^{\prime \prime}
$$

where $\overline{\boldsymbol{\Sigma}}_{k}$ are defined in (106) and (107), respectively. The model (114) is identical to the parallel channel model (22)-(24) with the size of sets $\mathcal{A}, \mathcal{B}$, and $\mathcal{C}$ in (108)-(110) and with a noise power that is larger by a factor of $\sigma^{2} / \bar{s}^{2}$. Since a constant factor in the noise power does not affect the s.d.o.f., the coding schemes described in Section V-A achieve the lower bound in Theorem 1.

\section{CONCLUSION}

In this paper, we have studied the two-transmitter Gaussian complex MIMO-MAC wiretap channel where the eavesdropper channel is arbitrarily varying and its state is known to the eavesdropper only, and the main channel is static and its state is known to all nodes. We have completely characterized the s.d.o.f. region for this channel for all possible antenna configurations. We have proved that this s.d.o.f. region can be achieved by a scheme that orthogonalizes the transmit signals of the two users at the intended receiver, in which each user achieves secrecy guarantee independently without cooperation from the other user. The converse was proved by carefully changing the set of signals available to the eavesdropper through an induction procedure in order to obtain an upper bound on a weighted-sum-rate expression.

We note that the scope of this paper is limited to the case of two-transmitters. Our proof involves simultaneously decomposing the channel matrices of the two users into parallel channels using the GSVD. Then a set of eavesdropper channels is carefully constructed for the parallel-channel model to obtain an upper bound, tighter than the usual cut-set bound. Since the GSVD transform does not easily extend to more than two matrices, we did not pursue the case of more than two transmitters and leave this extension as a future work. We also note that our setup assumes that the channel matrices of the legitimate receivers are static, i.e., fixed for the entire period of communication. Our core ideas readily extend to the case when the channel gains of the legitimate users change over time, but are revealed to all the terminals.

As suggested by this paper, the optimal strategy for a communication network where the eavesdropper channel is arbitrarily varying can potentially be very different from the case where the eavesdropper channel is fixed and its state is known to all terminals. This is also observed for example in the MIMO broadcast channel [21] and the two-way channel [38], [39].

Finally, we note that the proposed setup allows the eavesdropper terminals to perfectly emulate the legitimate receiver's channel if sufficiently many antennas are available. Such an assumption may be unavoidable if the environment is uncontrolled and an eavesdropper could be placed right where the intended receiver is located. In controlled environments where the eavesdropper must maintain a certain physical separation, our proposed setup may still be realistic if not pessimistic.
APPENDIX A PROOF OF LEMMA 1

For $R_{s, 1}$, from Fano's inequality, we have

$$
\begin{aligned}
n\left(R_{s, 1}-\delta_{n}\right) & \leq I\left(W_{1} ; Y_{\mathcal{A} \cup \mathcal{B} \cup \mathcal{C}}^{n}\right)-I\left(W_{1} ; Y_{\mathcal{E}_{1}}^{n}\right) \\
& \leq I\left(W_{1} ; Y_{\mathcal{A} \cup \mathcal{B} \cup C}^{n} \mid Y_{\mathcal{E}_{1}}^{n}\right) \\
& \leq I\left(W_{1} ; Y_{\mathcal{A} \cup \mathcal{B} \cup \mathcal{C}}^{n}, X_{2, \mathcal{B} \cup \mathcal{C}}^{n} \mid Y_{\mathcal{E}_{1}}^{n}\right) \\
& =I\left(W_{1} ; Y_{\mathcal{A} \cup \mathcal{B}}^{n}, X_{2, \mathcal{B} \cup \mathcal{C}}^{n} \mid Y_{\mathcal{E}_{1}}^{n}\right)
\end{aligned}
$$

where the last step (118) relies on the fact that the additive noise at each receiver end of each subchannel in Fig. 5 is independent from each other and hence

$$
Y_{\mathcal{C}}^{n} \rightarrow X_{2, \mathcal{C}}^{n} \rightarrow\left(W_{1}, Y_{\mathcal{A} \cup \mathcal{B}}^{n}, Y_{\mathcal{E}_{1}}^{n}, X_{2, \mathcal{B}}^{n}\right)
$$

holds. Since $\left(X_{2, \mathcal{C}}^{n}, X_{2, \mathcal{B}}^{n}\right)$ is independent from $W_{1}$ and $\mathcal{E}_{1} \subseteq \mathcal{A}$, (118) can be written as

$$
\begin{aligned}
& I\left(W_{1} ; Y_{\mathcal{A} \cup \mathcal{B}}^{n} \mid Y_{\mathcal{E}_{1}}^{n}, X_{2, \mathcal{B} \cup \mathcal{C}}^{n}\right) \\
= & I\left(W_{1} ; Y_{\left(\mathcal{A} \backslash \mathcal{E}_{1}\right) \cup \mathcal{B}}^{n} \mid Y_{\mathcal{E}_{1}}^{n}, X_{2, \mathcal{B} \cup \mathcal{C}}^{n}\right) \\
= & I\left(W_{1} ; Y_{\mathcal{A} \backslash \mathcal{E}_{1}}^{n} \mid Y_{\mathcal{E}_{1}}^{n}, X_{2, \mathcal{B} \cup \mathcal{C}}^{n}\right)+I\left(W_{1} ; Y_{\mathcal{B}}^{n} \mid Y_{\mathcal{A}}^{n}, X_{2, \mathcal{B} \cup \mathcal{C}}^{n}\right)
\end{aligned}
$$

where the last step (120) follows from the fact $\mathcal{E}_{1} \subseteq \mathcal{A}$ and hence $\mathcal{A}=\left(\mathcal{A} \backslash \mathcal{E}_{1}\right) \cup \mathcal{E}_{1}$. We separately bound each of the two terms above

$$
\begin{aligned}
& I\left(W_{1} ; Y_{\mathcal{A} \backslash \mathcal{E}_{1}}^{n} \mid Y_{\mathcal{E}_{1}}^{n}, X_{2, \mathcal{B} \cup \mathcal{C}}^{n}\right) \\
& \leq I\left(W_{1}, Y_{\mathcal{E}_{1}}^{n}, X_{2, \mathcal{B} \cup \mathcal{C}}^{n} ; Y_{\mathcal{A} \backslash \mathcal{E}_{1}}^{n}\right) \\
& \leq I\left(W_{1}, Y_{\mathcal{E}_{1}}^{n}, X_{2, \mathcal{B} \cup \mathcal{C}}^{n}, X_{1, \mathcal{A} \backslash \mathcal{E}_{1}}^{n} ; Y_{\mathcal{A} \backslash \mathcal{E}_{1}}^{n}\right) \\
& =I\left(X_{1, \mathcal{A} \backslash \mathcal{E}_{1}}^{n} ; Y_{\mathcal{A} \backslash \mathcal{E}_{1}}^{n}\right)
\end{aligned}
$$

where the last step follows from the Markov chain relation $Y_{\mathcal{A} \backslash \mathcal{E}_{1}}^{n} \leftrightarrow X_{\mathcal{A} \backslash \mathcal{E}_{1}}^{n} \leftrightarrow\left(W_{1}, Y_{\mathcal{E}_{1}}^{n}, X_{2, \mathcal{B} \cup \mathcal{C}}^{n}\right)$. We upper bound the second term in (120) as follows:

$$
\begin{aligned}
I & \left(W_{1} ; Y_{\mathcal{B}}^{n} \mid Y_{\mathcal{A}}^{n}, X_{2, \mathcal{B} \cup \mathcal{C}}^{n}\right) \\
\leq & I\left(X_{1, \mathcal{A} \cup \mathcal{B}}^{n} ; Y_{\mathcal{B}}^{n} \mid Y_{\mathcal{A}}^{n}, X_{2, \mathcal{B} \cup \mathcal{C}}^{n}\right) \\
= & I\left(X_{1, \mathcal{B}}^{n} ; Y_{\mathcal{B}}^{n} \mid Y_{\mathcal{A}}^{n}, X_{2, \mathcal{B} \cup \mathcal{C}}^{n}\right) \\
& +I\left(X_{1, \mathcal{A}}^{n} ; Y_{\mathcal{B}}^{n} \mid Y_{\mathcal{A}}^{n}, X_{2, \mathcal{B} \cup \mathcal{C}}^{n}, X_{1, \mathcal{B}}^{n}\right) \\
& =I\left(X_{1, \mathcal{B}}^{n} ; Y_{\mathcal{B}}^{n} \mid Y_{\mathcal{A}}^{n}, X_{2, \mathcal{B} \cup \mathcal{C}}^{n}\right)
\end{aligned}
$$

where we use the Markov relation $W_{1} \leftrightarrow X_{1, \mathcal{A} \cup \mathcal{B}}^{n} \leftrightarrow$ $\left(Y_{\mathcal{A}}^{n}, X_{2, \mathcal{B} \cup \mathcal{C}}^{n}\right)$ in step (124) and (126) follows from the fact Markov relation

$$
Y_{\mathcal{B}}^{n} \leftrightarrow\left(X_{1, \mathcal{B}}^{n}, X_{2, \mathcal{B}}^{n}\right) \leftrightarrow\left(X_{2, \mathcal{C}}^{n}, Y_{\mathcal{A}}^{n}\right) .
$$

Note that (41) follows upon substituting (123) and (126) into (120). 
For $R_{s, 2}$, from Fano's inequality and the secrecy constraint, we have

$$
\begin{aligned}
& n\left(R_{s, 2}-\delta_{n}\right) \leq I\left(W_{2} ; Y_{\mathcal{A} \cup \mathcal{B} \cup \mathcal{C}}^{n}\right)-I\left(W_{2} ; X_{2, \mathcal{E}_{2}}^{n}\right) \\
\leq & I\left(W_{2} ; Y_{\mathcal{A} \cup \mathcal{B} \cup \mathcal{C}}^{n} \mid X_{2, \mathcal{E}_{2}}^{n}\right) \\
= & I\left(W_{2} ; Y_{\mathcal{B} \cup \mathcal{C}}^{n} \mid Y_{\mathcal{A}}^{n}, X_{2, \mathcal{E}_{2}}^{n}\right) \\
= & I\left(W_{2} ; Y_{\left(\mathcal{C} \backslash \mathcal{E}_{2}\right) \cup \mathcal{B}}^{n} \mid Y_{\mathcal{A}}^{n}, X_{2, \mathcal{E}_{2}}^{n}\right) \\
= & I\left(W_{2} ; Y_{\mathcal{C} \backslash \mathcal{E}_{2}}^{n} \mid Y_{\mathcal{A}}^{n}, X_{2, \mathcal{E}_{2}}^{n}\right)+I\left(W_{2} ; Y_{\mathcal{B}}^{n} \mid Y_{\mathcal{A}}^{n}, X_{2, \mathcal{E}_{2}}^{n}, Y_{\mathcal{C} \backslash \mathcal{E}_{2}}^{n}\right)
\end{aligned}
$$

where (130) follows from the fact that $Y_{\mathcal{A}}^{n}$ is independent of $\left(W_{2}, X_{2, \mathcal{B} \cup \mathcal{C}}^{n}\right)$ and (131) follows from the fact that $Y_{\mathcal{E}_{2}}^{n} \rightarrow X_{2, \mathcal{E}_{2}}^{n} \rightarrow\left(Y_{\mathcal{B} \cup \mathcal{C} \backslash \mathcal{E}_{2}}^{n}, W_{2}, Y_{\mathcal{A}}^{n}\right)$ holds. We separately bound each term in (132)

$$
\begin{aligned}
& I\left(W_{2} ; Y_{\mathcal{C} \backslash \mathcal{E}_{2}}^{n} \mid Y_{\mathcal{A}}^{n}, X_{2, \mathcal{E}_{2}}^{n}\right) \leq I\left(W_{2}, Y_{\mathcal{A}}^{n}, X_{2, \mathcal{E}_{2}}^{n} ; Y_{\mathcal{C} \backslash \mathcal{E}_{2}}^{n}\right) \\
& \leq I\left(X_{2, \mathcal{C} \backslash \mathcal{E}_{2}}^{n}, W_{2}, Y_{\mathcal{A}}^{n}, X_{2, \mathcal{E}_{2}}^{n} ; Y_{\mathcal{C} \backslash \mathcal{E}_{2}}^{n}\right) \\
& =I\left(X_{2, \mathcal{C} \backslash \mathcal{E}_{2}}^{n} ; Y_{\mathcal{C} \backslash \mathcal{E}_{2}}^{n}\right)
\end{aligned}
$$

where the justification for establishing (135) is identical to (123) and hence omitted. We finally bound the second term in (132)

$$
\begin{aligned}
& I\left(W_{2} ; Y_{\mathcal{B}}^{n} \mid Y_{\mathcal{A}}^{n}, X_{2, \mathcal{E}_{2}}^{n}, Y_{C \backslash \mathcal{E}_{2}}^{n}\right) \\
\leq & I\left(X_{2, \mathcal{B} \cup \mathcal{C}}^{n} ; Y_{\mathcal{B}}^{n} \mid Y_{\mathcal{A}}^{n}, X_{2, \mathcal{E}_{2}}^{n}, Y_{\mathcal{C} \backslash \mathcal{E}_{2}}^{n}\right) \\
\leq & I\left(Y_{\mathcal{A}}^{n}, X_{2, \mathcal{B} \cup \mathcal{C}}^{n}, X_{2, \mathcal{E}_{2}}^{n}, Y_{\mathcal{C} \backslash \mathcal{E}_{2}}^{n} ; Y_{\mathcal{B}}^{n}\right) \\
= & I\left(Y_{\mathcal{A}}^{n}, X_{2, \mathcal{B} \cup \mathcal{C}}^{n}, X_{2, \mathcal{E}_{2}}^{n} ; Y_{\mathcal{B}}^{n}\right) \\
& +I\left(Y_{\mathcal{C} \backslash \mathcal{E}_{2}}^{n} ; Y_{\mathcal{B}}^{n} \mid Y_{\mathcal{A}}^{n}, X_{2, \mathcal{B} \cup \mathcal{C}}^{n}, X_{2, \mathcal{E}_{2}}^{n}\right) \\
= & I\left(Y_{\mathcal{A}}^{n}, X_{2, \mathcal{B} \cup \mathcal{C}}^{n} ; Y_{\mathcal{B}}^{n}\right)
\end{aligned}
$$

where the justification for arriving at (140) is similar to (126) and hence omitted.

Substituting (135) and (140) into (132), we establish (42).

\section{APPENDIX B}

ProOF OF LEMMA 2

Assume the eavesdropper monitors $Y_{\mathcal{A}}^{n}$ and $X_{1, \mathcal{E}_{1} \backslash \mathcal{A}}^{n}$ for $W_{1}$. Then for $R_{s, 1}$, from Fano's inequality, we have

$$
\begin{aligned}
& n\left(R_{s, 1}-\delta_{n}\right) \\
\leq & I\left(W_{1} ; Y_{\mathcal{A} \cup \mathcal{B} \cup \mathcal{C}}^{n}\right)-I\left(W_{1} ; Y_{\mathcal{A}}^{n}, X_{1, \mathcal{E}_{1} \backslash \mathcal{A}}^{n}\right) \\
\leq & I\left(W_{1} ; Y_{\mathcal{A} \cup \mathcal{B} \cup \mathcal{C}}^{n} \mid Y_{\mathcal{A}}^{n}, X_{1, \mathcal{E}_{1} \backslash \mathcal{A}}^{n}\right) \\
= & I\left(W_{1} ; Y_{\mathcal{B} \cup \mathcal{C}}^{n} \mid Y_{\mathcal{A}}^{n}, X_{1, \mathcal{E}_{1} \backslash \mathcal{A}}^{n}\right) \\
\leq & I\left(W_{1} ; Y_{\mathcal{B} \cup \mathcal{C}}^{n}, X_{2, \mathcal{B} \cup \mathcal{C}}^{n} \mid Y_{\mathcal{A}}^{n}, X_{1, \mathcal{E}_{1} \backslash \mathcal{A}}^{n}\right) \\
= & I\left(W_{1} ; Y_{\mathcal{B} \cup \mathcal{C}}^{n} \mid Y_{\mathcal{A}}^{n}, X_{1, \mathcal{E}_{1} \backslash \mathcal{A}}^{n}, X_{2, \mathcal{B} \cup \mathcal{C}}^{n}\right) \\
= & I\left(W_{1} ; Y_{\mathcal{F}}^{n} \mid Y_{\mathcal{A}}^{n}, X_{1, \mathcal{E}_{1} \backslash \mathcal{A}}^{n}, X_{2, \mathcal{B} \cup \mathcal{C}}^{n}\right) \\
= & I\left(W_{1} ; Y_{\mathcal{F}}^{n} \mid Y_{\mathcal{A}}^{n}, X_{1, \mathcal{B} \backslash \mathcal{F}}^{n}, X_{2, \mathcal{B} \cup \mathcal{C}}^{n}\right)
\end{aligned}
$$

where (145) follows from the fact that $X_{2, \mathcal{B} \cup \mathcal{C}}^{n}$ is independent of $\left(W_{1}, Y_{\mathcal{A}}^{n}, X_{1, \mathcal{E}_{1} \backslash \mathcal{A}}^{n}\right)$, while (146) follows from the fact that since the noise across the channels is independent the Markov condition

$$
\left(Y_{\mathcal{E}_{1} \backslash \mathcal{A}}^{n}, Y_{\mathcal{C}}^{n}\right) \leftrightarrow\left(X_{1, \mathcal{E}_{1} \backslash \mathcal{A}}^{n}, X_{2, \mathcal{B} \cup \mathcal{C}}^{n}\right) \leftrightarrow\left(W_{1}, Y_{B \backslash \mathcal{E}_{1}}^{n}, Y_{\mathcal{A}}^{n}\right)
$$

holds and furthermore we have defined $\mathcal{F}=\mathcal{B} \backslash \mathcal{E}_{1}$.

Since the channel noise is independent of the message, $W_{1} \leftrightarrow$ $X_{1, \mathcal{A} \cup \mathcal{B}}^{n} \leftrightarrow\left(Y_{\mathcal{F} \cup \mathcal{A}}^{n}, X_{1, \mathcal{B} \backslash \mathcal{F}}^{n}, X_{2, \mathcal{B} \cup \mathcal{C}}^{n}\right)$ holds. Hence,

$$
\begin{aligned}
& I\left(W_{1} ; Y_{\mathcal{F}}^{n} \mid Y_{\mathcal{A}}^{n}, X_{1, \mathcal{B} \backslash \mathcal{F}}^{n}, X_{2, \mathcal{B} \cup \mathcal{C}}^{n}\right) \\
\leq & I\left(X_{1, \mathcal{A} \cup \mathcal{B}}^{n} ; Y_{\mathcal{F}}^{n} \mid Y_{\mathcal{A}}^{n}, X_{1, \mathcal{B} \backslash \mathcal{F}}^{n}, X_{2, \mathcal{B} \cup \mathcal{C}}^{n}\right) \\
= & I\left(X_{1, \mathcal{F}}^{n} ; Y_{\mathcal{F}}^{n} \mid Y_{\mathcal{A}}^{n}, X_{1, \mathcal{B} \backslash \mathcal{F}}^{n}, X_{2, \mathcal{B} \cup \mathcal{C}}^{n}\right) \\
& \quad+I\left(X_{1, \mathcal{A} \cup \mathcal{B} \backslash \mathcal{F}}^{n} ; Y_{\mathcal{F}}^{n} \mid Y_{\mathcal{A}}^{n}, X_{1, \mathcal{B}}^{n}, X_{2, \mathcal{B} \cup \mathcal{C}}^{n}\right) \\
= & I\left(X_{1, \mathcal{F}}^{n} ; Y_{\mathcal{F}}^{n} \mid Y_{\mathcal{A}}^{n}, X_{1, \mathcal{B} \backslash \mathcal{F}}^{n}, X_{2, \mathcal{B} \cup \mathcal{C}}^{n}\right)
\end{aligned}
$$

where the last step uses the fact that the second term in (150) involves conditioning on $\left(X_{1, \mathcal{F}}^{n}, X_{2, \mathcal{F}}^{n}\right)$ and hence is zero. This establishes (48).

For $R_{s, 2}$, we assume the eavesdropper is monitoring $X_{2, \mathcal{C}}^{n}, X_{2 . \mathcal{E}_{2} \backslash \mathcal{C}}^{n}$ for $W_{2}$. Using Fano's inequality and the secrecy constraint, we have

$$
\begin{aligned}
& n\left(R_{s, 2}-\delta_{n}\right) \leq I\left(W_{2} ; Y_{\mathcal{A} \cup \mathcal{B} \cup \mathcal{C}}^{n}\right)-I\left(W_{2} ; X_{2, \mathcal{E}_{2}}^{n}\right) \\
\leq & I\left(W_{2} ; Y_{\mathcal{A} \cup \mathcal{B} \cup \mathcal{C}}^{n} \mid X_{2, \mathcal{E}_{2}}^{n}\right) \\
\leq & I\left(W_{2} ; Y_{\mathcal{A} \cup \mathcal{B} \cup \mathcal{C}}^{n}, X_{1, \mathcal{E}_{2} \cap \mathcal{B}}^{n} \mid X_{2, \mathcal{E}_{2}}^{n}\right) \\
= & I\left(W_{2} ; Y_{\mathcal{B} \cup \mathcal{C}}^{n} \mid X_{2, \mathcal{E}_{2}}^{n}, Y_{\mathcal{A}}^{n}, X_{1, \mathcal{E}_{2} \cap \mathcal{B}}^{n}\right) \\
\leq & I\left(X_{2, \mathcal{B} \cup \mathcal{C}}^{n} ; Y_{\mathcal{B} \cup \mathcal{C}}^{n} \mid X_{2, \mathcal{E}_{2}}^{n}, Y_{\mathcal{A}}^{n}, X_{1, \mathcal{E}_{2} \cap \mathcal{B}}^{n}\right) \\
= & I\left(X_{2, \mathcal{B} \cup \mathcal{C}}^{n} ; Y_{\mathcal{G} \cup \mathcal{E}_{2}}^{n} \mid X_{2, \mathcal{E}_{2}}^{n}, Y_{\mathcal{A}}^{n}, X_{1, \mathcal{E}_{2} \cap \mathcal{B}}^{n}\right) \\
= & I\left(X_{2, \mathcal{B} \cup \mathcal{C}}^{n} ; Y_{\mathcal{G}}^{n} \mid X_{2, \mathcal{E}_{2}}^{n}, Y_{\mathcal{A}}^{n}, X_{1, \mathcal{E}_{2} \cap \mathcal{B}}^{n}\right) \\
& \quad+I\left(X_{2, \mathcal{B} \cup \mathcal{C}}^{n} ; Y_{\mathcal{E}_{2}}^{n} \mid X_{2, \mathcal{E}_{2}}^{n}, Y_{\mathcal{A} \cup \mathcal{G}}^{n}, X_{1, \mathcal{E}_{2} \cap \mathcal{B}}^{n}\right) \\
= & I\left(X_{2, \mathcal{B} \cup \mathcal{C}}^{n} ; Y_{\mathcal{G}}^{n} \mid X_{2, \mathcal{E}_{2}}^{n}, Y_{\mathcal{A}}^{n}, X_{1, \mathcal{E}_{2} \cap \mathcal{B}}^{n}\right) \\
\leq & I\left(X_{2, \mathcal{B} \cup \mathcal{C}}^{n}, Y_{\mathcal{A}}^{n}, X_{1, \mathcal{E}_{2} \cap \mathcal{B}}^{n} ; Y_{\mathcal{G}}^{n}\right) \\
\leq & I\left(M, X_{1, \mathcal{B} \backslash \mathcal{G}}^{n} ; Y_{\mathcal{G}}^{n}\right)
\end{aligned}
$$

where (155) follows from the fact that $\left(X_{1 \mathcal{E}_{2} \cap \mathcal{B}}^{n}, Y_{\mathcal{A}}^{n}\right)$ are the transmitted signals from user 1 and independent of $\left(W_{2}, X_{2, \mathcal{E}_{2}}^{n}\right)$ and (157) follows from the fact that $\mathcal{C} \subseteq \mathcal{E}_{2} \subseteq \mathcal{B} \cup \mathcal{C}$ and $\mathcal{G}=\mathcal{B} \backslash \mathcal{E}_{2}$ and hence $\mathcal{E}_{2} \cup \mathcal{G}=\mathcal{B} \cup \mathcal{C}$ holds. Equation (159) follows from the fact that since the noise on each channel is Markov, we have $Y_{\mathcal{E}_{2}}^{n} \leftrightarrow\left(X_{2, \mathcal{E}_{2}}^{n}, X_{1, \mathcal{E}_{2} \cap \mathcal{B}}^{n}\right) \leftrightarrow\left(Y_{\mathcal{A} \cup \mathcal{G}}^{n}, X_{\mathcal{B} \cup \mathcal{C}}^{n}\right)$ and hence the second term in (158) is zero.

Hence, we have proved Lemma 2.

\section{REFERENCES}

[1] C. E. Shannon, "Communication theory of secrecy systems," Bell Syst. Tech. J., vol. 28, no. 4, pp. 656-715, Sep. 1949.

[2] I. Csiszár and J. Körner, "Broadcast channels with confidential messages," IEEE Trans. Inf. Theory, vol. 24, no. 3, pp. 339-348, May 1978.

[3] Y. Liang, H. Poor, and S. Shamai Shitz, "Information theoretic security," Found. Trends Commun. Inf. Theory, vol. 5, no. 4-5, pp. 355-580, 2009. 
[4] M. Bloch and J. Barros, Physical-Layer Security: From Information Theory to Security Engineering. Cambridge, U.K.: Cambridge Univ. Press, 2011.

[5] A. Thangaraj, S. Dihidar, A. R. Calderbank, S. McLaughlin, and J. Merolla, "On the application of LDPC codes to a novel wiretap channel inspired by quantum key distribution," IEEE Trans. Inf. Theory, vol. 53, no. 8, pp. 2933-2945, Aug. 2005.

[6] M. Bellare and S. Tessaro, Polynomial-time, semantically-secure encryption achieving the secrecy capacity 2012 [Online]. Available: http://arxiv.org/abs/1201.3160

[7] X. Li, J. Hwu, and E. P. Ratazzi, "Using antenna array redundancy and channel diversity for secure wireless transmissions," J. Commun., vol. 2, no. 3, pp. 24-32, 2007.

[8] S. Goel and R. Negi, "Guaranteeing secrecy using artificial noise," IEEE Trans. Wireless Commun., vol. 7, no. 6, pp. 2180-2189, Jun. 2008.

[9] A. Khisti, G. W. Wornell, A. Wiesel, and Y. Eldar, "On the Gaussian MIMO wiretap channel," in Proc. IEEE Int. Symp. Inf. Theory, Jun. 2007, pp. 2471-2475

[10] A. Khisti and G. Wornell, "Secure transmission with multiple antennas-I: The MISOME wiretap channel," IEEE Trans. Inf. Theory, vol. 56, no. 7, pp. 3088-3104, Jul. 2010.

[11] A. Khisti and G. Wornell, "Secure transmission with multiple antennas-II: The MIMOME wiretap channel," IEEE Trans. Inf. Theory, vol. 56, no. 11, pp. 5515-5532, Nov. 2010.

[12] T. Liu and S. S. Shamai, "A note on the secrecy capacity of the multiple-antenna wiretap channel," IEEE Trans. Inf. Theory, vol. 55, no. 6, pp. 2547-2553, Jun. 2009.

[13] F. Oggier and B. Hassibi, "The secrecy capacity of the MIMO wiretap channel," IEEE Trans. Inf. Theory, vol. 57, no. 8, pp. 4961-4972, Aug. 2011.

[14] S. Shafiee, N. Liu, and S. Ulukus, "Towards the secrecy capacity of the Gaussian MIMO wire-tap channel: The 2-2-1 channel," IEEE Trans. Inf. Theory, vol. 55, no. 9, pp. 4033-4039, Sep. 2009.

[15] R. Liu, T. Liu, and H. V. Poor, "Multiple-input multiple-output Gaussian broadcast channels with confidential messages," IEEE Trans. Inf. Theory, vol. 56, no. 9, pp. 4215-4227, Sep. 2010.

[16] E. Ekrem and S. Ulukus, "The secrecy capacity region of the Gaussian MIMO multi-receiver wiretap channel," IEEE Trans. Inf. Theory, vol. 57, no. 4, pp. 2083-2114, Apr. 2011

[17] M. Kobayashi, Y. Liang, S. Shamai, and M. Debbah, "On the compound MIMO broadcast channels with confidential messages," in Proc. IEEE Int. Symp. Inf. Theory, Jun. 2009, pp. 1283-1287.

[18] A. Mukherjee and A. L. Swindlehurst, "Robust beamforming for security in MIMO wiretap channels with imperfect CSI," IEEE Trans. Signal Process., vol. 59, no. 1, pp. 351-361, Jan. 2011.

[19] E. MolavianJazi, "Secure communication over arbitrarily varying wiretap channels," Master thesis, , Dec. 2009 [Online]. Available: http://etd.nd.edu/ETD-db/theses/available/etd-12112009-112419/unrestricted/MolavianJaziE122009.pdf

[20] X. He and A. Yener, "MIMO wiretap channels with arbitrarily varying eavesdropper channel states," Jul. 2010 [Online]. Available: http://arxiv.org/abs/1007.4801

[21] X. He, A. Khisti, and A. Yener, "MIMO broadcast channel with arbitrarily varying eavesdropper channel: Secrecy degrees of freedom," in Proc. IEEE Global Telecommun. Conf., Dec. 2011, pp. 1-5.

[22] E. Tekin and A. Yener, "The general Gaussian multiple access and two-way wire-tap channels: Achievable rates and cooperative jamming," IEEE Trans. Inf. Theory, vol. 54, no. 6, pp. 2735-2751, Jun. 2008.

[23] Y. Liang and H. V. Poor, "Multiple access channels with confidential messages," IEEE Trans. Inf. Theory, vol. 54, no. 3, pp. 976-1002, Mar. 2008.

[24] E. Ekrem and S. Ulukus, "On the secrecy of multiple access wiretap channel," in Proc. 46th Annu. Allerton Conf. Commun., Control, Comput., Sep. 2008, pp. 1014-1021.

[25] D. S. Papailiopoulos and A. G. Dimakis, "Distributed storage codes meet multiple-access wiretap channels," in Proc. 48th Allerton Conf. Commun., Control Comput., Sep. 2010.

[26] X. He and A. Yener, "Providing secrecy with structured codes: Tools and applications to Gaussian two-user channels," Jul. 2009 [Online]. Available: http://arxiv.org/abs/0907.5388
[27] G. Bagherikaram, A. S. Motahari, and A. K. Khandani, "On the secure DoF of the single-antenna MAC," in Proc. IEEE Int. Symp. Inf. Theory, Jun. 2010, pp. 2588-2592.

[28] X. He, "Cooperation and Information Theoretic Security in Wireless Networks," PhD dissertation, , Aug. 2010 [Online]. Available: http://etda.libraries.psu.edu/theses/approved/WorldWideIndex/ETD5342/index.html

[29] J. Xie and S. Ulukus, "Secure degrees of freedom of one-hop wireless networks," Sep. 2012 [Online]. Available: http://arxiv.org/abs/1209. 5370

[30] Y. Liang, G. Kramer, H. V. Poor, and S. Shamai, "Compound wiretap channels," Eurasip J. Wireless Commun. Netw.-Spec. Issue Wireless Phys. Layer Security, vol. 2009, 2009, article ID 142374.

[31] S. Yang, P. Piantanida, M. Kobayashi, and S. Shamai, "On the secrecy degrees of freedom of multi-antenna wiretap channels with delayed CSIT," in Proc. IEEE Int. Symp. Inf. Theory, 2011, pp. 2866-2870.

[32] I. Csiszar and J. Körner, Information Theory: Coding Theorems for Discrete Memoryless Systems. Cambridge, U.K.: Cambridge Univ. Press, 2011.

[33] A. Khisti, "Interference alignment for the multi-antenna compound wiretap channel," IEEE Trans. Inf. Theory, vol. 57, no. 5, pp. 2967-2993, May 2011.

[34] D. N. C. Tse and S. V. Hanly, "Multiaccess fading channels. I. Polymatroid structure, optimal resource allocation and throughput capacities," IEEE Trans. Inf. Theory, vol. 44, no. 7, pp. 2796-2815, Nov. 1998.

[35] C. C. Paige and M. A. Saunders, "Towards a generalized singular value decomposition," SIAM J. Numer. Anal., vol. 18, no. 3, pp. 398-405, 1981.

[36] A. Khisti, D. Silva, and F. Kschischang, "Secure broadcast codes over linear deterministic channels," in Proc. IEEE Int. Symp. Inf. Theory Process., May 2010, pp. 555-559.

[37] E. Ekrem and S. Ulukus, "Degrees of freedom region of the Gaussian MIMO broadcast channel with common and private messages," in Proc. IEEE Global Telecommun. Conf., Dec. 2010, pp. 1-5.

[38] X. He and A. Yener, "Secrecy when the eavesdropper controls its channel states," in Proc. IEEE Int. Symp. Inf. Theory Process., Jul. 2011, pp. 618-622.

[39] X. He and A. Yener, "Gaussian two-way wiretap channel with an arbitrarily varying eavesdropper," in Proc. IEEE GLOBECOM Workshops, Dec. 2011, pp. 854-858.

Xiang He (S'08-M'10) received the B.S. and M.S. degrees in electrical engineering from Shanghai Jiao Tong University, Shanghai, China in 2003 and 2006, respectively. His master study is about high speed FPGA implementation of channel encoder, decoder and MIMO detectors. He received the Ph.D degree in 2010 from the Department of Electrical Engineering at the Pennsylvania State University and joined Microsoft in that year. In 2010, he received Melvin P. Bloom Memorial Outstanding Doctoral Research Award from the Department of Electrical Engineering at the Pennsylvania State University and the best paper award from the Communication Theory Symposium in IEEE International Conference on Communications (ICC). In 2011, he was named as one of the exemplary reviewers by IEEE Communication Letters. His research interests include information theoretic secrecy, coding theory, queuing theory, optimization techniques, distributed detection and estimation.

Ashish Khisti (M'08) is an Assistant Professor in the Electrical and Computer Engineering (ECE) Department and a Canada Research Chair (Tier II) in Network Information Theory at the University of Toronto, Toronto, Ontario, Canada. He received his BASc degree in Engineering Sciences from University of Toronto in 2002 and his S.M. and Ph.D. degrees from the Massachusetts Institute of Technology (MIT), Cambridge, MA, USA in 2004 and 2008, respectively. He has been with the University of Toronto since 2009. His research interests span the areas of information theory, wireless physical layer security and streaming communication systems. During his graduate studies, Professor Khisti was a recipient of the NSERC postgraduate fellowship, Harold H. Hazen Teaching award and the Morris Joseph Levin Masterworks award. At the University of Toronto he is a recipient of the Ontario Early Researcher Award (2012) and a Hewlett-Packard IRP award (2011, 2012). He is an associate editor of the IEEE TRANSACTIONS ON COMMUNICATIONS.

Professor Khisti co-organized a workshop on Physical Layer Security at IEEE GLOBECOM Conference (2011) and a workshop on Interactive Information Theory at the Banff International Research Station (2012). 
Aylin Yener (S'91-M'00) received the B.Sc. degree in electrical and electronics engineering, and the B.Sc. degree in physics, from Boğaziçi University, Istanbul, Turkey; and the M.S. and Ph.D. degrees in electrical and computer engineering from Wireless Information Network Laboratory (WINLAB), Rutgers University, New Brunswick, NJ. Commencing fall 2010, for three semesters, she was a P.C. Rossin Assistant Professor at the Electrical Engineering and Computer Science Department, Lehigh University, PA. In 2002, she joined the faculty of The Pennsylvania State University, University Park, PA, where she was an Assistant Professor, then Associate Professor, and is currently Professor of Electrical Engineering since 2010. During the academic year 2008-2009, she was a Visiting Associate Professor with the Department of Electrical Engineering, Stanford University, CA, USA. Her research interests are in information theory, communication theory and network science, with recent emphasis on green communications and information security. She received the NSF CAREER award in 2003.

Dr. Yener served as the student committee chair for the IEEE Information Theory Society 2007-2011, and was the co-founder of the Annual School of Information Theory in North America co-organizing the school in 2008, 2009 and 2010. She currently serves on the board of governors as the treasurer of the IEEE Information Theory Society. 